OPEN ACCESS

Edited by:

Min Ye,

Peking University, China

Reviewed by:

Zhishan Ding,

Zhejiang Chinese Medical University,

China

Shuai Ji,

Xuzhou Medical University,

China

*Correspondence:

Jishuang Chen

biochenjs@njtech.edu.cn

tThese authors have contributed equally to this work

Specialty section:

This article was submitted to

Ethnopharmacology,

a section of the journal

Frontiers in Pharmacology

Received: 16 April 2019 Accepted: 11 September 2019 Published: 01 November 2019

Citation:

Xu D, Pan Y and Chen J (2019) Chemical Constituents, Pharmacologic Properties, and Clinical Applications of Bletilla striata.

Front. Pharmacol. 10:1168. doi: 10.3389/fphar.2019.01168

\section{Chemical Constituents, Pharmacologic Properties, and Clinical Applications of Bletilla striata}

\author{
Delin Xu't, Yinchi Pan ${ }^{1 \dagger}$ and Jishuang Chen ${ }^{2 *}$ \\ ${ }^{1}$ Department of Cell Biology, Zunyi Medical University, Zunyi, China, ${ }^{2}$ Key Laboratory of Basic Pharmacology and Joint \\ International Research Laboratory of Ethnomedicine of Ministry of Education, Zunyi Medical University, Zunyi, China
}

Bletilla striata is a plant from the Orchidaceae family that has been employed as a traditional Chinese medicine (TCM) for thousands of years in China. Here, we briefly review the published studies of the last 30 years that were related to chemical constituents, pharmacologic activities, and clinical applications of B. striata. Approximately 158 compounds have been extracted from $B$. striata tubers with clarified molecular structures that were classified as glucosides, bibenzyls, phenanthrenes, quinones, biphenanthrenes, dihydrophenanthrenes, anthocyanins, steroids, triterpenoids, and phenolic acids. These chemicals support the pharmacological properties of hemostasis and wound healing, and also exhibit anti-oxidation, anti-cancer, anti-viral, and anti-bacterial activities. Additionally, various clinical trials conducted on $B$. striata have demonstrated its marked activities as an embolizing and mucosa-protective agent, and its application for use in novel biomaterials, quality control, and toxicology. It also has been widely used as a constituent of many preparations in TCM formulations, but because there are insufficient studies on its clinical properties, its efficacy and safety cannot be established from a scientific point of view. We hope that this review will provide reference for further research and development of this unique plant.

Keywords: Bletilla striata, chemical constituents, pharmacological activities, clinical application, quality control, toxicology

\section{INTRODUCTION}

Bletilla striata (Thunb.) Rchb. f. (Orchidaceae), also known as Bletillae Rhizoma, is considered to be merely an ornamental plant in Europe and the USA. However, B. striata, which is widely distributed in China, Japan, Korea, Mongolia, and Myanmar, is important because of its use in traditional Chinese medicine (TCM).

According to the earliest pharmacopeia of TCM, Shennong's Materia Medica Classic, Chinese scholars were the first to describe the morphologic features and medicinal value of $B$. striata (Hossain, 2011; Chen et al., 2018). Other Chinese pharmacopeias have recorded the effect of its astringency upon hemostasis and analgesia, as well as its use for treating traumatic bleeding, ulcers, and swelling and chapped skin (He et al., 2016). Physicians in Korea and Japan have used B. striata to treat tuberculosis, whooping cough, bleeding of the stomach/duodenal ulcers, abscesses, swellings, and parasitic diseases (Rhee et al., 1982). Several studies have revealed its uses in TCM, its chemical constituents, and medicinal properties (Yang et al., 2019). 
This review summarizes current knowledge regarding the chemical composition, bioactivities, and pharmacologic effects of $B$. striata. We also provide a brief summary to provide insights into the TCM-based uses of B. striata, and a scientific basis for developing new medicines utilizing this interesting plant.

\section{CHEMICAL CONSTITUENTS}

The study of the chemical constituents of $B$. striata can be traced back to the late 19th century, but Japanese scholars began systematic research only in 1983 (Takagi et al., 1983). Studies have shown that the chemical constituents of $B$. striata are mainly glucosides, bibenzyls, phenanthrenes, quinones, biphenanthrenes, dihydrophenanthrenes, anthocyanins, steroids, triterpenoids, and phenolic acids. Their names (1-158) appear in Table 1, and their structures (1-158) are shown in Figures 1-11. These chemical components form the material basis for the medicinal value of B. striata.

\section{Glycosides}

Glycosides are an important class of compounds in B. striata (Figure 1), of which polysaccharides are relatively abundant in its dried tubers. B. striata contains various natural monosaccharide compounds (1-19). Bletilla striata polysaccharide (BSP) is a high-molecular-weight, viscous polysaccharide, whose chemical composition is glucomannan (which comprises D-glucose and D-mannose) (Liu et al., 2013).

\section{Bibenzyls}

Although the skeleton of bibenzyl compounds is simple, the bridge chain substituents attached to the aromatic ring are diverse. This scenario leads to various structural types and, thus, to various biologic activities (Dai and Sun, 2008). The structure of bibenzyl compounds is unique, and this is of considerable practical importance for finding benzyl compounds with potential medicinal value. B. striata is rich in benzyl compounds (20-47), containing 28 types, as shown in Figure 2.

\section{Phenanthrenes}

Several studies have reported that phenanthrenes are present in B. striata. The substituents on the aromatic rings are mainly methoxy, hydroxyl, and $p$-hydroxybenzyl groups, as shown in Figure 3.

\section{Quinones}

Benzoquinones, naphthoquinones, anthraquinones, and phenanthraquinones are commonly found in plants used for TCM. Five types of quinones have been isolated from B. striata, including anthraquinones (67-68) and phenanthraquinones (69-71), whose structures are shown in Figure 4.

\section{Biphenanthrenes}

Due to their axial asymmetry and asymmetric induction, biphenanthrene compounds exhibit important characteristic absorption in the infrared region at $1620-1480 \mathrm{~cm}^{-1}$ and 900 $650 \mathrm{~cm}^{-1}$ (Tang et al., 2014). The biphenanthrene compounds in B. striata are composed of two simple phenanthrenes or dihydrophides, as shown in Figure 5.

\section{Dihydrophenanthrenes}

Dihydrophenanthrenes, phenanthrenes, and benzyls are stilbenoids with an identical 1,2-distyrene skeleton. The substituents on their aromatic rings are mainly hydroxyl, methoxy, and p-hydroxybenzyl groups (Lin et al., 2005). In the ultraviolet spectrum, dihydrophenanthrene compounds, in general, have an $\alpha$ band at approximately $310 \mathrm{~nm}$, a $\beta$ band at approximately $250 \mathrm{~nm}$, and a $\rho$ band at approximately $280 \mathrm{~nm}$. The skeleton of dihydrophenanthrenes can be synthesized in the phenylpropane metabolic pathway using phenolic compounds (Gu et al., 2013). The structures of dihydrophenanthrenes are shown in Figure 6.

\section{Anthocyanins}

Anthocyanins are the main pigments that give flowers their color. The flowers of B. striata are not commonly used in TCM, and therefore, few studies have been conducted on these flowers. Only five compounds (113-117) have been isolated from the flowers of B. striata. However, recent studies have shown that the anthocyanins in plants may affect human health (Stintzing and Carle, 2004), and have provided new material to study the medicinal uses of $B$. striata. The structures of anthocyanins are shown in Figure 7.

\section{Steroids}

All steroids are biosynthesized from mevalonic acid (Debieu et al., 1992). The steroids present in B. striata are complex, with diverse structures and many derivatives. Eleven steroids and their derivatives (118-128) have been isolated from B. striata, and their structures are shown in Figure 8.

\section{Triterpenoids}

Triterpenoids are widespread in plants. They are mostly tetracyclic and pentacyclic, but a few are monocyclic, bicyclic, and tricyclic (Liou and Wu, 2002). The triterpenoids isolated from B. striata have mainly tetracyclic triterpene structures (129-135), but also include a pentacyclic triterpene branched diglycoside compound (136). Their structures are shown in Figure 9.

\section{Phenolic Acids}

Phenolic acids are organic acids containing phenol rings, hydroxybenzoic acids, and hydroxycinnamic acids. They are important secondary metabolites in plants because of the protection they offer against insects, viruses, and bacteria (Heleno et al., 2015). Consumption of phenolic acid-rich food could assist with accelerating the elimination of oxygen free radicals in the body, which would protect cells (Bae et al., 2016). Twelve phenolic acids (137-148) have been obtained from $B$. striata, and their structures are shown in Figure 10. 
TABLE 1 | List of 158 compounds isolated from B. striata.

\begin{tabular}{|c|c|c|c|c|}
\hline No. & Compound Name & Chemical Formula & Plant Part & References \\
\hline \multicolumn{5}{|c|}{ Glycosides } \\
\hline 1 & dactylorhin A & $\mathrm{C}_{40} \mathrm{H}_{56} \mathrm{O}_{22}$ & tubers & (Feng et al., 2008) \\
\hline 3 & gymnoside I & $\mathrm{C}_{21} \mathrm{H}_{30} \mathrm{O}_{11}$ & tubers & (Feng et al., 2008) \\
\hline 4 & gymnoside II & $\mathrm{C}_{21} \mathrm{H}_{30} \mathrm{O}_{11}$ & tubers & (Feng et al., 2008) \\
\hline 5 & gymnoside V & $\mathrm{C}_{49} \mathrm{H}_{62} \mathrm{O}_{23}$ & tubers & (Yan et al., 2014) \\
\hline 6 & gymnoside IX & $\mathrm{C}_{51} \mathrm{H}_{64} \mathrm{O}_{24}$ & tubers & (Yan et al., 2014) \\
\hline 7 & gymnoside $X$ & $\mathrm{C}_{51} \mathrm{H}_{64} \mathrm{O}_{24}$ & tubers & (Yan et al., 2014) \\
\hline 8 & militarine & $\mathrm{C}_{34} \mathrm{H}_{46} \mathrm{O}_{17}$ & tubers & (Han et al., 2002b) \\
\hline 9 & bletilnoside A & $\mathrm{C}_{38} \mathrm{H}_{62} \mathrm{O}_{12}$ & roots & (Park et al., 2014) \\
\hline 10 & bletilnoside B & $\mathrm{C}_{38} \mathrm{H}_{60} \mathrm{O}_{12}$ & roots & (Park et al., 2014) \\
\hline 11 & 3-O- $\beta$-D-glucopyranosyl-3-epiruscogenin & $\mathrm{C}_{41} \mathrm{H}_{60} \mathrm{O}_{12}$ & roots & (Park et al., 2014) \\
\hline 12 & 3-O- $\beta$-D-glucopyranosyl-3-epineoruscogenin & $\mathrm{C}_{41} \mathrm{H}_{58} \mathrm{O}_{12}$ & roots & (Park et al., 2014) \\
\hline 13 & dancosterol & $\mathrm{C}_{35} \mathrm{H}_{60} \mathrm{O}_{6}$ & tubers & (Han et al., 2001) \\
\hline 14 & 2,7-dihydroxy-4-methoxyphenanthrene-2-O-glucoside & $\mathrm{C}_{21} \mathrm{H}_{22} \mathrm{O}_{8}$ & tubers & (Yamaki et al., 1993b) \\
\hline 15 & 2,7-dihydroxy-4-methoxyphenanthrene-2,7-O-diglucoside & $\mathrm{C}_{27} \mathrm{H}_{32} \mathrm{O}_{13}$ & tubers & (Yamaki et al., 1993b) \\
\hline 16 & 3,7-dihydroxy-2,4-dimethoxyphenanthrene-3-O-glucoside & $\mathrm{C}_{22} \mathrm{H}_{24} \mathrm{O}_{9}$ & tubers & (Yamaki et al., 1993b) \\
\hline 17 & gastrodin & $\mathrm{C}_{13} \mathrm{H}_{18} \mathrm{O}_{7}$ & fibrous roots & (Yu et al., 2016) \\
\hline 18 & $\begin{array}{l}\text { 2,7-dihydroxy-l-(4'-hydroxybenzyl)-9,10-dihydrophenan-threne- } \\
\text { 4'-O-glucoside }\end{array}$ & $\mathrm{C}_{28} \mathrm{H}_{30} \mathrm{O}_{9}$ & tubers & (Yamaki et al., 1993b) \\
\hline 19 & 3'-hydroxy-5-methoxybibenzyl-3-O- $\beta$-D-glucopyranoside & $\mathrm{C}_{21} \mathrm{H}_{26} \mathrm{O}_{8}$ & tubers & (Han et al., 2002b) \\
\hline \multicolumn{5}{|c|}{ Bibenzyls } \\
\hline 20 & blestritin A & $\mathrm{C}_{37} \mathrm{H}_{36} \mathrm{O}_{6}$ & tubers & (Feng et al., 2008) \\
\hline 21 & blestritin B & $\mathrm{C}_{30} \mathrm{H}_{30} \mathrm{O}_{6}$ & tubers & (Feng et al., 2008) \\
\hline 22 & blestritin C & $\mathrm{C}_{36} \mathrm{H}_{34} \mathrm{O}_{6}$ & tubers & (Feng et al., 2008) \\
\hline 23 & bulbocodin & $\mathrm{C}_{36} \mathrm{H}_{34} \mathrm{O}_{6}$ & tubers & (Feng et al., 2008) \\
\hline 24 & bulbocodin C & $\mathrm{C}_{29} \mathrm{H}_{28} \mathrm{O}_{5}$ & tubers & (Ma et al., 2017) \\
\hline 25 & bulbocodin D & $\mathrm{C}_{29} \mathrm{H}_{28} \mathrm{O}_{5}$ & tubers & (Feng et al., 2008) \\
\hline 26 & bulbocol & $\mathrm{C}_{23} \mathrm{H}_{24} \mathrm{O}_{4}$ & tubers & (Feng et al., 2008) \\
\hline 27 & gymconopin D & $\mathrm{C}_{23} \mathrm{H}_{24} \mathrm{O}_{4}$ & tubers & (Feng et al., 2008) \\
\hline 28 & shancigusin B & $\mathrm{C}_{28} \mathrm{H}_{26} \mathrm{O}_{5}$ & tubers & (Ma et al., 2017) \\
\hline 29 & shanciguol & $\mathrm{C}_{28} \mathrm{H}_{26} \mathrm{O}_{5}$ & tubers & (Ma et al., 2017) \\
\hline 30 & arundinan & $\mathrm{C}_{22} \mathrm{H}_{22} \mathrm{O}_{3}$ & tubers & (Ma et al., 2017) \\
\hline 31 & arundin & $\mathrm{C}_{29} \mathrm{H}_{28} \mathrm{O}_{4}$ & tubers & (Ma et al., 2017) \\
\hline 32 & batatasin III & $\mathrm{C}_{15} \mathrm{H}_{16} \mathrm{O}_{3}$ & tubers & (Woo et al., 2014) \\
\hline 33 & gigantol & $\mathrm{C}_{16} \mathrm{H}_{18} \mathrm{O}_{4}$ & tubers & (Woo et al., 2014) \\
\hline 34 & 3,4'-dihydroxy-5,3',5'-trimethoxybibenzyl & $\mathrm{C}_{17} \mathrm{H}_{20} \mathrm{O}_{5}$ & tubers & (Woo et al., 2014) \\
\hline 35 & 3,3'-dihydroxy-5,4'-dimethoxybibenzyl & $\mathrm{C}_{16} \mathrm{H}_{18} \mathrm{O}_{4}$ & tubers & (Feng et al., 2008) \\
\hline 36 & 3'-O-methylbatatasin III & $\mathrm{C}_{15} \mathrm{H}_{18} \mathrm{O}_{3}$ & tubers & (Feng et al., 2008) \\
\hline 37 & 3,3'-dihydroxy-4-(p-hydroxybenzyl)-5-methoxybibenzyl & $\mathrm{C}_{22} \mathrm{H}_{22} \mathrm{O}_{4}$ & tubers & (Bai et al., 1993) \\
\hline 38 & 3,3'-dihydroxy-2-(p-hydroxybenzyl)-5-methoxybibenzyl & $\mathrm{C}_{22} \mathrm{H}_{22} \mathrm{O}_{4}$ & tubers & (Bai et al., 1993) \\
\hline 39 & 3',5-dihydroxy-2-(p-hydroxybenzyl)-3-methoxybibenzyl & $\mathrm{C}_{22} \mathrm{H}_{22} \mathrm{O}_{4}$ & tubers & (Bai et al., 1993) \\
\hline 40 & 2',6'-bis(p-hydroxybenzyl)-5-methoxybibenzyl-3,3'-diol & $\mathrm{C}_{33} \mathrm{H}_{36} \mathrm{O}_{5}$ & tubers & (Takagi et al., 1983) \\
\hline 41 & 2,6-bis(p-hydroxybenzyl)-5,3'-dimethoxybibenzyl-3-ol & $\mathrm{C}_{30} \mathrm{H}_{30} \mathrm{O}_{5}$ & tubers & (Takagi et al., 1983) \\
\hline 42 & 3,3'-dihydroxy-5-methoxy-2,5',6-tris(p -hydroxybenzyl) bibenzyl & $\mathrm{C}_{46} \mathrm{H}_{44} \mathrm{O}_{11}$ & tubers & (Takagi et al., 1983) \\
\hline 43 & 3,3',5-trimethoxybibenzyl & $\mathrm{C}_{17} \mathrm{H}_{20} \mathrm{O}_{3}$ & tubers & (Yamaki et al., 1991) \\
\hline 44 & 3,5-dimethoxybibenzyl & $\mathrm{C}_{16} \mathrm{H}_{18} \mathrm{O}_{2}$ & tubers & (Yamaki et al., 1991) \\
\hline 45 & 5-hydroxy-4-(p-hydroxybenzyl)-3',3-dimethoxybibenzyl & $\mathrm{C}_{23} \mathrm{H}_{24} \mathrm{O}_{4}$ & tubers & (Han et al., 2002a) \\
\hline 46 & 3,3'-dihydroxy-5-methoxybibenzyl & $\mathrm{C}_{15} \mathrm{H}_{16} \mathrm{O}_{3}$ & tubers & (Han et al., 2002c) \\
\hline 47 & 5-hydroxy-2-(p-hydroxybenzyl)-3-methoxybibenzyl & $\mathrm{C}_{22} \mathrm{H}_{22} \mathrm{O}_{3}$ & tubers & (Ma et al., 2017) \\
\hline \multicolumn{5}{|c|}{ Phenanthrenes } \\
\hline 48 & 4-methoxyphenanthrene-2,7-diol & $\mathrm{C}_{15} \mathrm{H}_{12} \mathrm{O}_{3}$ & tubers & (Feng et al., 2008) \\
\hline 49 & 3,4-dimethoxyphenanthrene-2,7-diol & $\mathrm{C}_{16} \mathrm{H}_{14} \mathrm{O}_{4}$ & tubers & (Feng et al., 2008) \\
\hline 50 & 2,4-dimethoxyphenanthrene-3,7-diol & $\mathrm{C}_{16} \mathrm{H}_{14} \mathrm{O}_{4}$ & tubers & (Feng et al., 2008) \\
\hline 51 & 3,5-dimethoxyphenanthrene-2,7-diol & $\mathrm{C}_{16} \mathrm{H}_{14} \mathrm{O}_{4}$ & tubers & (Xiao et al., 2016) \\
\hline 52 & 1,5-dimethoxyphenanthrene-2,7-diol & $\mathrm{C}_{16} \mathrm{H}_{14} \mathrm{O}_{4}$ & tubers & (Xiao et al., 2016) \\
\hline 53 & 2,4-dimethoxyphenanthrene-7-ol & $\mathrm{C}_{15} \mathrm{H}_{14} \mathrm{O}_{3}$ & tubers & (Xiao et al., 2016) \\
\hline 54 & 2,4,7-trimethoxyphenanthrene & $\mathrm{C}_{17} \mathrm{H}_{16} \mathrm{O}_{3}$ & tubers & (Yamaki et al., 1991) \\
\hline 55 & 2,3,4,7-tetramethoxyphenanthrene & $\mathrm{C}_{18} \mathrm{H}_{18} \mathrm{O}_{4}$ & tubers & (Yamaki et al., 1991) \\
\hline 56 & 1,8-bis(p-hydroxybenzyl)-4-methoxyphenanthrene-2,7-diol & $\mathrm{C}_{29} \mathrm{H}_{24} \mathrm{O}_{5}$ & tubers & (Bai et al., 1991) \\
\hline 57 & 1-(p-hydroxybenzyl)-4,8-dimethoxyphenanthrene-2,7-diol & $\mathrm{C}_{23} \mathrm{H}_{20} \mathrm{O}_{5}$ & tubers & (Morita et al., 2005) \\
\hline 58 & 1-(p-hydroxybenzyl)-4-methoxyphenanthrene-2,7-diol & $\mathrm{C}_{22} \mathrm{H}_{18} \mathrm{O}_{4}$ & tubers & (Yamaki et al., 1990) \\
\hline 59 & 2-hydroxy-4,7-dimethoxyphenanthrene & $\mathrm{C}_{16} \mathrm{H}_{14} \mathrm{O}_{3}$ & fibrous roots & (Yu et al., 2016) \\
\hline 60 & 3,7-dihydroxy-2,4,8-trimethoxyphenanthrene & $\mathrm{C}_{17} \mathrm{H}_{16} \mathrm{O}_{5}$ & tubers & (Woo et al., 2014) \\
\hline
\end{tabular}

(Continued) 
TABLE 1 | Continued

\begin{tabular}{|c|c|c|c|c|}
\hline No. & Compound Name & Chemical Formula & Plant Part & References \\
\hline 61 & 2,7-dihydroxy-3,4-dimethoxyphenanthrene & $\mathrm{C}_{16} \mathrm{H}_{14} \mathrm{O}_{4}$ & tubers & (Han et al., 2002c) \\
\hline 62 & 1-(p-hydroxybenzyl)-4,7-dimethoxyphenanthrene-2-ol & $\mathrm{C}_{23} \mathrm{H}_{20} \mathrm{O}_{4}$ & tubers & (Xiao et al., 2017) \\
\hline 63 & 1-(p-hydroxybenzyl)-4,7-dimethoxyphenanthrene-2,8-diol & $\mathrm{C}_{23} \mathrm{H}_{20} \mathrm{O}_{5}$ & tubers & (Xiao et al., 2017) \\
\hline 64 & 1-(p-hydroxybenzyl)-4,7-dimethoxyphenanthrene-2,6-diol & $\mathrm{C}_{23} \mathrm{H}_{20} \mathrm{O}_{5}$ & tubers & (Xiao et al., 2017) \\
\hline 65 & bleformin B & $\mathrm{C}_{23} \mathrm{H}_{20} \mathrm{O}_{5}$ & tubers & (Ma et al., 2017) \\
\hline 66 & blespirol & $\mathrm{C}_{25} \mathrm{H}_{18} \mathrm{O}_{5}$ & tubers & (Yamaki et al., 1993b) \\
\hline \multicolumn{5}{|c|}{ Quinones } \\
\hline 67 & 1,8-dihydroxy-3-methoxy-6-methylanthracene-9,10-dione & $\mathrm{C}_{16} \mathrm{H}_{12} \mathrm{O}_{5}$ & tubers & (Wang et al., 2001) \\
\hline 68 & 2-methylanthraquinone & $\mathrm{C}_{15} \mathrm{H}_{10} \mathrm{O}_{2}$ & tubers & (Sun et al., 2016c) \\
\hline 69 & 4,7-dimethoxyphenanthrene-1,2-dione & $\mathrm{C}_{16} \mathrm{H}_{13} \mathrm{O}_{4}$ & tubers & (Xiao et al., 2016) \\
\hline 70 & 7-hydroxy-2-methoxyphenanthrene-3,4-dione & $\mathrm{C}_{15} \mathrm{H}_{13} \mathrm{O}_{4}$ & tubers & (Sun et al., 2016a) \\
\hline 71 & 3',7',7-trihydroxy-2,2',4'-trimethoxy-[1,8'-biphenanthrene]-3,4-dione & $\mathrm{C}_{31} \mathrm{H}_{23} \mathrm{O}_{8}$ & tubers & (Sun et al., 2016a) \\
\hline \multicolumn{5}{|c|}{ Biphenanthrenes } \\
\hline 72 & blestrin A & $\mathrm{C}_{30} \mathrm{H}_{26} \mathrm{O}_{6}$ & tubers & (Bai et al., 1990) \\
\hline 73 & blestrin B & $\mathrm{C}_{30} \mathrm{H}_{26} \mathrm{O}_{6}$ & tubers & (Bai et al., 1990) \\
\hline 74 & blestrin C & $\mathrm{C}_{30} \mathrm{H}_{24} \mathrm{O}_{6}$ & tubers & (Yamaki et al., 1992) \\
\hline 75 & blestrin D & $\mathrm{C}_{30} \mathrm{H}_{24} \mathrm{O}_{6}$ & tubers & (Yamaki et al., 1992) \\
\hline 76 & blestriarene A & $\mathrm{C}_{30} \mathrm{H}_{26} \mathrm{O}_{6}$ & tubers & (Yamaki et al., 1989) \\
\hline 77 & blestriarene B & $\mathrm{C}_{30} \mathrm{H}_{24} \mathrm{O}_{6}$ & tubers & (Yamaki et al., 1989) \\
\hline 78 & blestriarene C & $\mathrm{C}_{30} \mathrm{H}_{22} \mathrm{O}_{6}$ & tubers & (Yamaki et al., 1989) \\
\hline 79 & blestrianol A & $\mathrm{C}_{30} \mathrm{H}_{26} \mathrm{O}_{6}$ & tubers & (Bai et al., 1991) \\
\hline 80 & blestrianol B & $\mathrm{C}_{37} \mathrm{H}_{32} \mathrm{O}_{7}$ & tubers & (Bai et al., 1991) \\
\hline 81 & blestrianol C & $\mathrm{C}_{37} \mathrm{H}_{30} \mathrm{O}_{7}$ & tubers & (Bai et al., 1991) \\
\hline 82 & $\begin{array}{l}\text { 4,7,3'5'-tetramethoxy-9',10'-dihydro-[1,2'-biphenanthrene]-2,7'- } \\
\text { diol }\end{array}$ & $\mathrm{C}_{32} \mathrm{H}_{27} \mathrm{O}_{6}$ & fibrous roots & (Qian et al., 2015) \\
\hline 83 & $\begin{array}{l}4,7,7 \text { '-trimethoxy-9',10'-dihydro-[1,3'-biphenanthrene]-2,2',5'- } \\
\text { triol }\end{array}$ & $\mathrm{C}_{31} \mathrm{H}_{25} \mathrm{O}_{6}$ & fibrous roots & (Qian et al., 2015) \\
\hline 84 & $\begin{array}{l}4,7,4 \text { '-trimethoxy-9',10'-dihydro-[1,1'-biphenanthrene]-2,2',7'- } \\
\text { triol }\end{array}$ & $\mathrm{C}_{31} \mathrm{H}_{25} \mathrm{O}_{6}$ & fibrous roots & (Qian et al., 2015) \\
\hline 85 & $\begin{array}{l}4,7,3 \text { ', 5'-tetramethoxy-9',10'-dihydro-[1, 1'-biphenanthrene]- } \\
2,2^{\prime}, 7^{\prime} \text { '-triol }\end{array}$ & $\mathrm{C}_{32} \mathrm{H}_{27} \mathrm{O}_{7}$ & fibrous roots & (Qian et al., 2015) \\
\hline 86 & 4,8,4',8'-tetramethoxy-[1,1'-biphenanthrene]-2,7,2',7'-tetrol & $\mathrm{C}_{32} \mathrm{H}_{26} \mathrm{O}_{8}$ & fibrous roots & (Qian et al., 2015) \\
\hline 87 & bleformin D & $\mathrm{C}_{37} \mathrm{H}_{32} \mathrm{O}_{7}$ & tubers & (Ma et al., 2017) \\
\hline 88 & $\begin{array}{l}\text { 4,4'-dimethoxy-9,10-dihydro-[6,1'-biphenanthrene]-2,7,2',7'- } \\
\text { tetraol }\end{array}$ & $\mathrm{C}_{30} \mathrm{H}_{24} \mathrm{O}_{6}$ & tubers & (Ma et al., 2017) \\
\hline 89 & gymconopin C & $\mathrm{C}_{30} \mathrm{H}_{26} \mathrm{O}_{6}$ & tubers & (Ma et al., 2017) \\
\hline \multicolumn{5}{|c|}{ Dihydrophenanthrenes } \\
\hline 90 & 4,7-dihydroxy-2-methoxy-9,10-dihydrophenanthrene & $\mathrm{C}_{15} \mathrm{H}_{14} \mathrm{O}_{3}$ & tubers & (Yamaki et al., 1990) \\
\hline 91 & $\begin{array}{l}\text { 2,7-dihydroxy-3-(p-hydroxybenzyl)-4-methoxy-9,10- } \\
\text { dihydrophenanthrene }\end{array}$ & $\mathrm{C}_{22} \mathrm{H}_{20} \mathrm{O}_{4}$ & tubers & (Yamaki et al., 1990) \\
\hline 92 & $\begin{array}{l}\text { 4,7-dihydroxy-1-(p-hydroxybenzyl)-2-methoxy-9,10- } \\
\text { dihydrophenanthrene }\end{array}$ & $\mathrm{C}_{22} \mathrm{H}_{20} \mathrm{O}_{4}$ & tubers & (Yamaki et al., 1990) \\
\hline 93 & $\begin{array}{l}\text { 2,7-dihydroxy-1,6-bis(p-hydroxybenzyl)-4-methoxy-9,10- } \\
\text { dihydrophenanthrene }\end{array}$ & $\mathrm{C}_{29} \mathrm{H}_{26} \mathrm{O}_{5}$ & tubers & (Yamaki et al., 1990) \\
\hline 94 & $\begin{array}{l}\text { 2,7-dihydroxy-l,3-bis(p-hydroxybenzyl)-4-methoxy-9,10- } \\
\text { dihydrophenanthrene }\end{array}$ & $\mathrm{C}_{29} \mathrm{H}_{26} \mathrm{O}_{5}$ & tubers & (Bai et al., 1993) \\
\hline 95 & $\begin{array}{l}\text { 2,7-dihydroxy-I-(p-hydroxybenzyl)-4-methoxy-9,10- } \\
\text { dihydrophenanthrene }\end{array}$ & $\mathrm{C}_{22} \mathrm{H}_{20} \mathrm{O}_{4}$ & tubers & (Bai et al., 1993) \\
\hline 96 & 2,4,7-trimethoxy-9,10-dihydrophenanthrene & $\mathrm{C}_{17} \mathrm{H}_{18} \mathrm{O}_{3}$ & tubers & (Yamaki et al., 1991) \\
\hline 97 & 2,7-dihydroxy-4-methoxy-9,10-dihydrophenanthrene & $\mathrm{C}_{15} \mathrm{H}_{14} \mathrm{O}_{3}$ & tubers & (Han et al., 2002c) \\
\hline 98 & 4,5-dihydroxy-2-methoxy-9,10-dihydrophenanthrene & $\mathrm{C}_{15} \mathrm{H}_{14} \mathrm{O}_{3}$ & fibrous roots & (Yu et al., 2016) \\
\hline 99 & 2,8-dihydroxy-4,7-dimethoxy-9,10-dihydrophenanthrene & $\mathrm{C}_{15} \mathrm{H}_{14} \mathrm{O}_{3}$ & tubers & (Woo et al., 2014) \\
\hline 100 & $\begin{array}{l}\text { 2,8-dihydroxy-1-(p-hydroxybenzyl)-4,7-dimethoxy-9,10- } \\
\text { dihydrophenanthrene }\end{array}$ & $\mathrm{C}_{23} \mathrm{H}_{22} \mathrm{O}_{5}$ & tubers & (Woo et al., 2014) \\
\hline 101 & pleionesin C & $\mathrm{C}_{27} \mathrm{H}_{26} \mathrm{O}_{7}$ & rhizomes & (Li et al., 2008a) \\
\hline 102 & $\begin{array}{l}\text { (2,3-trans)-2-(4-hydroxy-3-methoxyphenyl)-3-hydroxymethyl-10- } \\
\text { methoxy-2,3,4,5-tetrahydro-phenanthro[2,1-b]furan-7-ol }\end{array}$ & $\mathrm{C}_{25} \mathrm{H}_{24} \mathrm{O}_{6}$ & rhizomes & (Li et al., 2008a) \\
\hline 103 & bleochranol A & $\mathrm{C}_{40} \mathrm{H}_{38} \mathrm{O}_{8}$ & rhizomes & (Li et al., 2008a) \\
\hline 104 & bleochranol B & $\mathrm{C}_{25} \mathrm{H}_{24} \mathrm{O}_{6}$ & rhizomes & (Li et al., 2008a) \\
\hline 105 & bleochranol C & $\mathrm{C}_{33} \mathrm{H}_{32} \mathrm{O}_{8}$ & rhizomes & (Li et al., 2008a) \\
\hline 106 & bleochranol D & $\mathrm{C}_{34} \mathrm{H}_{32} \mathrm{O}_{8}$ & rhizomes & (Li et al., 2008a) \\
\hline 107 & $\begin{array}{l}\text { (2,3-trans)-3-[2-hydroxy-6-(3-hydro-xyphenethyl)-4- } \\
\text { methoxybenzyl]-2-(4-hydroxy-3-methoxyphenyl)-10-methoxy- } \\
\text { 2,3,4,5-tetrahydrophenanthro[2,1-b]furan-7-ol }\end{array}$ & $\mathrm{C}_{40} \mathrm{H}_{38} \mathrm{O}_{8}$ & rhizomes & (Li et al., 2008a) \\
\hline
\end{tabular}


TABLE 1 | Continued

\begin{tabular}{|c|c|c|c|c|}
\hline No. & Compound Name & Chemical Formula & Plant Part & References \\
\hline 108 & shanciol & $\mathrm{C}_{25} \mathrm{H}_{24} \mathrm{O}_{6}$ & tubers & (Ma et al., 2017) \\
\hline 109 & bletlos A & $\mathrm{C}_{28} \mathrm{H}_{28} \mathrm{O}_{8}$ & tubers & (Yamaki et al., 1993a) \\
\hline 110 & bletlos B & $\mathrm{C}_{27} \mathrm{H}_{26} \mathrm{O}_{7}$ & tubers & (Yamaki et al., 1993a) \\
\hline 111 & bletlos C & $\mathrm{C}_{27} \mathrm{H}_{26} \mathrm{O}$ & tubers & (Yamaki et al., 1993a) \\
\hline 112 & blestriaren A & $\mathrm{C}_{30} \mathrm{H}_{26} \mathrm{O}_{6}$ & rhizomes & (Li et al., 2008a) \\
\hline \multicolumn{5}{|c|}{ Anthocyanins } \\
\hline 113 & Bletilla anthocyanin 1 & $\mathrm{C}_{75} \mathrm{H}_{81} \mathrm{O}_{40}$ & flowers & (Saito et al., 1995) \\
\hline 114 & Bletilla anthocyanin 2 & $\mathrm{C}_{72} \mathrm{H}_{79} \mathrm{O}_{37}$ & flowers & (Saito et al., 1995) \\
\hline 115 & Bletilla anthocyanin 3 & $\mathrm{C}_{75} \mathrm{H}_{81} \mathrm{O}_{43}$ & flowers & (Saito et al., 1995) \\
\hline 116 & Bletilla anthocyanin 4 & $\mathrm{C}_{72} \mathrm{H}_{79} \mathrm{O}_{40}$ & flowers & (Saito et al., 1995) \\
\hline 117 & $\begin{array}{l}\text { 3-O-( } \beta \text {-glucopyranoside)-7-O-[6-O-(4-O-(6-O-(4-O- }(\beta- \\
\text { glucopyranosyl)-trans-caffeoyl)- } \beta \text {-glucopyranosyl)-trans-caffeoyl)- } \\
\beta \text {-glucopyranoside] }\end{array}$ & $\mathrm{C}_{57} \mathrm{H}_{63} \mathrm{O}_{32}$ & flowers & $\begin{array}{l}\text { (Tatsuzawa et al., } \\
\text { 2010) }\end{array}$ \\
\hline \multicolumn{5}{|c|}{ Steroids } \\
\hline 118 & $\beta$-sitosterol & $\mathrm{C}_{29} \mathrm{H}_{50} \mathrm{O}$ & tubers & (Han et al., 2001) \\
\hline 119 & $\beta$-sitosterol palmitate & $\mathrm{C}_{45} \mathrm{H}_{80} \mathrm{O}_{2}$ & tubers & (Yamaki et al., 1997) \\
\hline 120 & stigmasterol & $\mathrm{C}_{29} \mathrm{H}_{48} \mathrm{O}$ & tubers & (Sun et al., 2016c) \\
\hline 121 & stigamasterol palmitate & $\mathrm{C}_{45} \mathrm{H}_{78} \mathrm{O}_{2}$ & tubers & (Yamaki et al., 1997) \\
\hline 122 & 3-epiruscogenin & $\mathrm{C}_{27} \mathrm{H}_{42} \mathrm{O}_{4}$ & roots & (Park et al., 2014) \\
\hline 123 & 3-epineoruscogenin & $\mathrm{C}_{27} \mathrm{H}_{40} \mathrm{O}_{4}$ & roots & (Park et al., 2014) \\
\hline 124 & $\begin{array}{l}\text { (20S,22R)-1 } \beta, 2 \beta, 3 \beta, 4 \beta, 5 \beta, 7 \alpha \text {-hexahydroxyspirost-25(27)-en-6- } \\
\text { one }\end{array}$ & $\mathrm{C}_{27} \mathrm{H}_{35} \mathrm{O}_{9}$ & roots & (Park et al., 2014) \\
\hline 125 & $\begin{array}{l}(1 \alpha, 3 \alpha) \text {-1-O-[( } \beta \text {-D-xylopyranosyl- }(1 \rightarrow 2)-\alpha \text {-L-rhamnopyranosyl)]-3- } \\
\text { O-D-glucopyranosyl-5 } \alpha \text {-spirostan }\end{array}$ & $\mathrm{C}_{44} \mathrm{H}_{71} \mathrm{O}_{17}$ & roots & $\begin{array}{l}\text { (Wang and Meng } \\
\text { 2015) }\end{array}$ \\
\hline 126 & $\begin{array}{l}(1 \alpha, 3 \alpha)-1-O-[(\beta-D-x y l o p y r a n o s y l-(1 \rightarrow 2)-\alpha \text {-L-rhamnopyranosyl) } \\
\text { oxy]-3-O-D-glucopyranosyl-25(27)-ene-5 } \alpha \text {-spirostan }\end{array}$ & $\mathrm{C}_{44} \mathrm{H}_{69} \mathrm{O}_{17}$ & roots & $\begin{array}{l}\text { (Wang and Meng } \\
\text { 2015) }\end{array}$ \\
\hline 127 & $\begin{array}{l}(1 \alpha, 3 \alpha)-1-O-[(\beta-D-x y l o p y r a n o s y l-(1 \rightarrow 2)-\alpha \text {-L-rhamnopyranosyl) } \\
\text { oxy]-epiruscogenin }\end{array}$ & $\mathrm{C}_{38} \mathrm{H}_{59} \mathrm{O}_{12}$ & roots & $\begin{array}{l}\text { (Wang and Meng } \\
\text { 2015) }\end{array}$ \\
\hline 128 & $\begin{array}{l}(1 \alpha, 3 \alpha)-1-O-[(\beta-D-x y l o p y r a n o s y l-(1 \rightarrow 2)-\alpha-L-r h a m n o p y r a n o s y l) \\
\text { oxy]-epineoruscogenin }\end{array}$ & $\mathrm{C}_{38} \mathrm{H}_{57} \mathrm{O}_{12}$ & roots & $\begin{array}{l}\text { (Wang and Meng } \\
\text { 2015) }\end{array}$ \\
\hline \multicolumn{5}{|c|}{ Triterpenoids } \\
\hline 129 & cyclomargenol & $\mathrm{C}_{32} \mathrm{H}_{54} \mathrm{O}$ & tubers & (Yamaki et al., 1997) \\
\hline 130 & cyclomargenone & $\mathrm{C}_{32} \mathrm{H}_{53} \mathrm{O}$ & tubers & (Yamaki et al., 1997) \\
\hline 131 & cycloneolitsol & $\mathrm{C}_{32} \mathrm{H}_{54} \mathrm{O}$ & tubers & (Yamaki et al., 1997) \\
\hline 132 & cyclobalanone & $\mathrm{C}_{32} \mathrm{H}_{53} \mathrm{O}$ & tubers & (Yamaki et al., 1997) \\
\hline 133 & 24-methylenecycloartanol palmitate & $\mathrm{C}_{47} \mathrm{H}_{81} \mathrm{O}_{2}$ & tubers & (Yamaki et al., 1997) \\
\hline 134 & cyclolaudenol & $\mathrm{C}_{31} \mathrm{H}_{51} \mathrm{O}$ & tubers & (Yang et al., 2014) \\
\hline 135 & cyclolaudenone & $\mathrm{C}_{31} \mathrm{H}_{50} \mathrm{O}$ & tubers & (Yang et al., 2014) \\
\hline 136 & 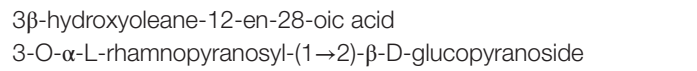 & $\mathrm{C}_{41} \mathrm{H}_{55} \mathrm{O}_{12}$ & tubers & (Sun et al., 2016b) \\
\hline \multicolumn{5}{|c|}{ Phenolic acids } \\
\hline 137 & p-hydroxybenzoic acid & $\mathrm{C}_{7} \mathrm{H}_{6} \mathrm{O}_{3}$ & tubers & (Takagi et al., 1983) \\
\hline 138 & protocatechuic acid & $\mathrm{C}_{7} \mathrm{H}_{6} \mathrm{O}_{4}$ & tubers & (Takagi et al., 1983) \\
\hline 139 & cinnamic acid & $\mathrm{C}_{9} \mathrm{H}_{8} \mathrm{O}_{2}$ & tubers & (Takagi et al., 1983) \\
\hline 140 & caffeic acid & $\mathrm{C}_{9} \mathrm{H}_{8} \mathrm{O}_{4}$ & tubers & (Han et al., 2001) \\
\hline 141 & 2-hydroxysuccinic acid & $\mathrm{C}_{4} \mathrm{H}_{5} \mathrm{O}_{5}$ & tubers & (Sun et al., 2016b) \\
\hline 142 & palmitic acid & $\mathrm{C}_{16} \mathrm{H}_{32} \mathrm{O}_{2}$ & tubers & (Sun et al., 2016b) \\
\hline 143 & syringaresinol & $\mathrm{C}_{22} \mathrm{H}_{26} \mathrm{O}_{8}$ & tubers & (Han et al., 2001) \\
\hline 144 & pinoresinol & $\mathrm{C}_{20} \mathrm{H}_{22} \mathrm{O}_{6}$ & tubers & (Bae et al., 2016) \\
\hline 145 & 3"-methoxynyasol & $\mathrm{C}_{18} \mathrm{H}_{17} \mathrm{O}_{3}$ & tubers & (Bae et al., 2016) \\
\hline 146 & p-hydroxybenzaldehyde & $\mathrm{C}_{7} \mathrm{H}_{6} \mathrm{O}_{2}$ & tubers & (Takagi et al., 1983) \\
\hline 147 & ferulic acid & $\mathrm{C}_{10} \mathrm{H}_{10} \mathrm{O}_{4}$ & tubers & (Yu et al., 2011) \\
\hline 148 & 3-hydroxycinnamic acid & $\mathrm{C}_{9} \mathrm{H}_{8} \mathrm{O}_{3}$ & tubers & (Yu et al., 2011) \\
\hline \multicolumn{5}{|c|}{ Others } \\
\hline 149 & 4-hydroxybenzylamine & $\mathrm{C}_{7} \mathrm{H}_{9} \mathrm{NO}$ & tubers & (Sun et al., 2016b) \\
\hline 150 & 4,4'-dihydroxydiphenylmethane & $\mathrm{C}_{13} \mathrm{H}_{12} \mathrm{O}_{2}$ & tubers & (Yan et al., 2014) \\
\hline 151 & 4,4'-dihydroxybenzyl sulfide & $\mathrm{C}_{14} \mathrm{H}_{14} \mathrm{SO}_{2}$ & tubers & (Yan et al., 2014) \\
\hline 152 & 5-(hydroxymethyl)-2-furaldehyde & $\mathrm{C}_{6} \mathrm{H}_{6} \mathrm{O}_{3}$ & tubers & (Sun et al., 2016c) \\
\hline 153 & striatolide & $\mathrm{C}_{18} \mathrm{H}_{30} \mathrm{O}_{3}$ & tubers & (Yan et al., 2014) \\
\hline 154 & schizandrin & $\mathrm{C}_{24} \mathrm{H}_{32} \mathrm{O}_{7}$ & tubers & (Han et al., 2002a) \\
\hline 155 & brugnanin & $\mathrm{C}_{56} \mathrm{H}_{90} \mathrm{O}_{8}$ & tubers & (Sun et al., 2016c) \\
\hline 156 & bletillanol A & $\mathrm{C}_{18} \mathrm{H}_{21} \mathrm{O}_{5}$ & tubers & (Bae et al., 2016) \\
\hline 157 & bletillanol B & $\mathrm{C}_{18} \mathrm{H}_{20} \mathrm{O}_{5}$ & tubers & (Bae et al., 2016) \\
\hline 158 & tupichinol A & $\mathrm{C}_{17} \mathrm{H}_{18} \mathrm{O}_{4}$ & tubers & (Bae et al., 2016) \\
\hline
\end{tabular}


<smiles>COc1ccc(COC(=O)CC(OC(=O)C(C)C)(C(=O)O)C(=O)OCc2ccc(OC)cc2)cc1</smiles><smiles>COC(=O)C(CC(=O)O)(OCc1ccc(OC(C)=O)cc1)C(C)C(C)C</smiles><smiles>COc1ccc(COC(=O)C(O)(CC(=O)O)C(C)C)cc1</smiles><smiles>CC(=O)Oc1ccc(COC(=O)CC(O)(CC(C)C)C(=O)O)cc1</smiles>

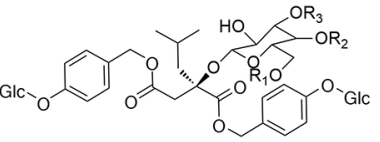

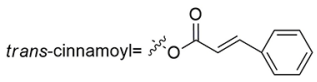

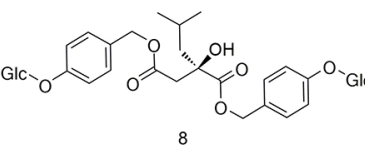

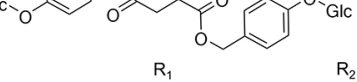
$\begin{array}{ll}6 & \text { Acetyl } \\ 7 & \text { Acetyl }\end{array}$ H trans-cinnamoyl trans-cinnamoyl $\mathrm{H}$

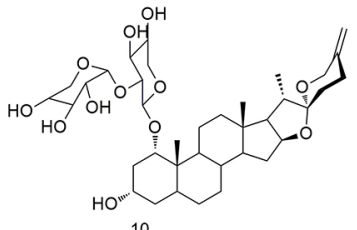

10

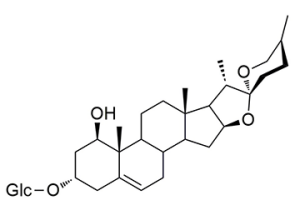
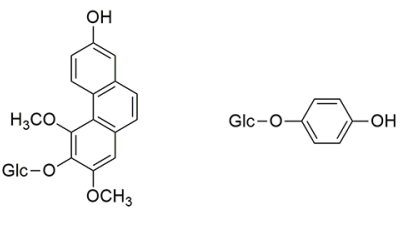

$15 \mathrm{Glc} \quad \mathrm{Gl}$

16

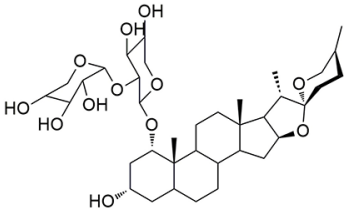

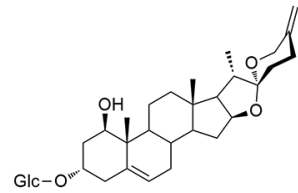

12

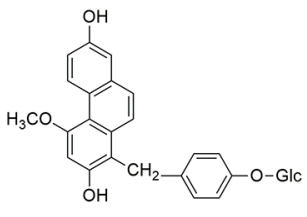

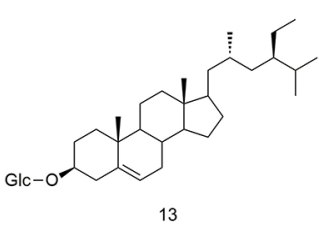

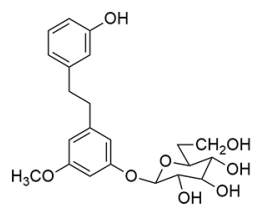

FIGURE 1 | Chemical structures of glycoside compounds (1-19) isolated from B. striata.

\section{Other Compounds}

In addition to the compounds stated above, other compounds from $B$. striata with different structures (149-158) have been reported. Their structures are shown in Figure 11.

\section{PHARMACOLOGIC ACTIVITIES}

The basic research of TCM focuses mainly on chemical composition and pharmacology, of which the latter is the most important. Pharmacologic studies on B. striata have mainly focused on pharmacokinetics. The latter advances our understanding of the mechanism of action of B. striata components. In recent years, pharmacologic studies on B. striata have focused mainly on its hemostatic, wound-healing, antioxidative, anti-cancer, antiviral, and antibacterial activities.

\section{Hemostasis}

Hemostasis is a process that causes bleeding to stop (i.e., blood is retained within a damaged blood vessel). The hemostatic effect is one of the main pharmacologic effects of B. striata. Bensky and colleagues showed that when the dried powder of B. striata tubers was mixed with water, it could achieve a good hemostatic effect after it was spread onto a wound (Venkatraja et al., 2012). Interestingly, recent studies have shown that the water-soluble portion of $B$. striata has an active role in hemostasis, and its function is believed to be related to adenosine diphosphate, which promotes and accelerates platelet aggregation ( $\mathrm{Lu}$ et al., 2005; Gachet, 2006). Hung and Wu found that BSP in this watersoluble component played a key role in hemostatic activity (Hung and $\mathrm{Wu}, 2016)$. Indeed, there has recently been intensive research on the procoagulant function of BSP.

Blood coagulation is a process in which a series of coagulation factors is successively activated by enzymatic action to produce thrombin and fibrinogen clots. Animal experiments have shown that BSP can participate in multiple hemostasis processes, such as platelet adherence to the subendothelial matrix to block vessels (primary hemostasis), and the formation of fibrin clots (secondary hemostasis) by activation of various coagulation factors and promotion of thromboxane-A2 synthesis (Dong et al., 2014). Because of its obvious and non-toxic hemostatic effect, BSP has been developed as a new type of hemostatic agent that can be used as a drug delivery vehicle and wound dressing (Zhang et al., 2017a). BSP can be combined with other materials to develop new biomedical materials, such as hemostatic materials for surgical treatments (Wang et al., 2017; Chen et al., 2019).

In addition to BSP, the steroids $(123,125-128)$ in $B$. striata exhibit obvious hemostatic activity and can significantly reduce clotting time (Yamaki et al., 1990). The hemostatic effect of this type of composition may be related to platelets, blood clotting, and fibrinolysis (Zhao et al., 2016). 


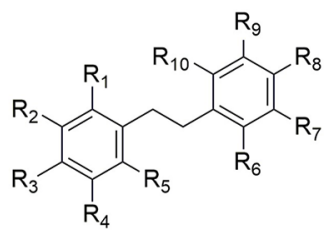

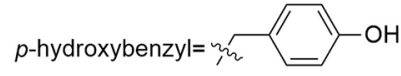

$\begin{array}{llllll}\mathrm{R}_{1} & \mathrm{R}_{2} & \mathrm{R}_{3} & \mathrm{R}_{4} & \mathrm{R}_{5} & \mathrm{R}_{6}\end{array}$

20

21

22

23

24

25

26

28

29

30

38

39

40

4

42

43

44

45

46

$47 \quad p$-hydroxybenzyl $\mathrm{OCH}$

p-hydroxybenzyl $\mathrm{OCH}_{3}$ p-hydroxybenzyl $\mathrm{H}$

p-hydroxybenzyl $\mathrm{OH}$

$\mathrm{H}$

$\mathrm{OCH}_{3}$ p-hydroxybenzyl $\mathrm{H}$

p-hydroxybenzyl $\mathrm{OH}$

$\mathrm{H}$

$\mathrm{H}$

$\mathrm{OCH}_{3}$ p-hydroxybenzyl $\mathrm{H}$

p-hydroxybenzyl $\mathrm{OH}$

$\mathrm{OCH}_{3}$ p-hydroxybenzyl $\mathrm{H}$

p-hydroxybenzyl $\mathrm{OCH}_{3}$ p-hydroxybenzyl $\mathrm{OH}$

p-hydroxybenzyl $\mathrm{OH}$ p-hydroxybenzyl $\mathrm{OCH}_{3}$

p-hydroxybenzyl $\mathrm{OCH}_{3}$

p-hydroxybenzyl $\mathrm{OH}$

p-hydroxybenzyl $\mathrm{OH}$

p-hydroxybenzyl $\mathrm{OH}$

p-hydroxybenzyl $\mathrm{OH}$

p-hydroxybenzyl $\mathrm{OH}$

$\mathrm{H} \quad \mathrm{OCH}_{3}$

$\mathrm{H}$

$\mathrm{OCH}_{3}$

$\mathrm{OCH}_{3}$

$\mathrm{OH}$

$\mathrm{OH}$

$\mathrm{OH} p$-hydroxybenzyl $\mathrm{OCH}_{3}$

$\mathrm{H}$

OH

p-hydroxybenzyl $\mathrm{OH}$

$\mathrm{H}$

$\mathrm{OCH}_{3}$

$\mathrm{H} \quad \mathrm{H}$

p-hydroxybenzyl $\mathrm{OCH}_{3}$

p-hydroxybenzyl $\mathrm{OCH}_{3}$

$\mathrm{H}$

$\mathrm{OH}$

$\mathrm{H}$

$\mathrm{H}$

$\mathrm{H}$

$\mathrm{H}$

\section{$\mathrm{H}$}

$\mathrm{H}$

(a)

$\mathrm{H}$

$\mathrm{H}$

$\mathrm{H}$

$\mathrm{OH} \quad$-hydroxybenzyl $\mathrm{H}$

$\begin{array}{lll}\mathrm{OCH}_{3} & \mathrm{H} & \mathrm{H}\end{array}$

$\mathrm{OCH}_{3}$ p-hydroxybenzyl $\mathrm{H}$

$\begin{array}{lll}\mathrm{OH} & \mathrm{H}\end{array}$

$\mathrm{OH}$

$\mathrm{H}$

$\mathrm{OH}$

$\mathrm{H}$

$\mathrm{OCH}_{3} \quad \mathrm{H}$

$\mathrm{OCH}_{3} \quad \mathrm{H}$

p-hydroxybenzyl $\mathrm{OCH}_{3}$

$$
\begin{aligned}
& \mathrm{H} \\
& \mathrm{H}
\end{aligned}
$$

$\mathrm{OH}$

p-hydroxybenzyl $\mathrm{H}$

p-hydroxybenzyl $\mathrm{OCH}_{3}$

$\mathrm{H}$

$\mathrm{OH} \quad p$-hydroxybenzyl $\mathrm{H}$

$\mathrm{OH} \quad p$-hydroxybenzyl $\mathrm{H}$

$\mathrm{OCH}_{3} \quad \mathrm{H}$

$\mathrm{OCH}_{3}$

$\mathrm{H}$

$\mathrm{H}$

$\mathrm{OCH}_{3}$

$\mathrm{H}$

$\mathrm{OH} p$-hydroxybenzyl $\mathrm{OCH}_{3}$

$\mathrm{H}$

$\mathrm{H} \quad \mathrm{OH}$

$\mathrm{H} \quad \mathrm{OCH}_{3}$

$\mathrm{H}$

$\mathrm{OH}$
$\mathrm{H}$

FIGURE 2 | Chemical structures of bibenzyls (20-47) isolated from B. striata.

\section{Wound Healing}

Wound healing has three main phases: inflammation, proliferation of granulation tissue, and repair (Kasuya and Tokura, 2014). Antioxidants in plants, such as polysaccharides and phenolic compounds, have important roles in these three phases (Süntar et al., 2012).

In the inflammation phase, BSP promotes the expression of cytokines such as tumor necrosis factor (TNF)- $\alpha$, interleukin 

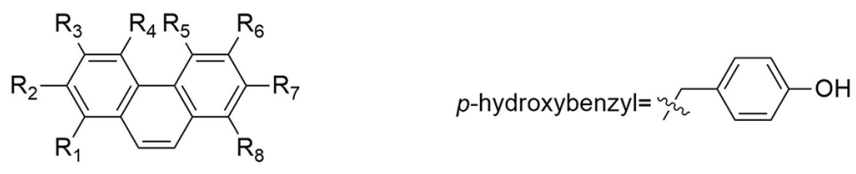

\begin{tabular}{|c|c|c|c|c|c|c|c|c|}
\hline & $\mathrm{R}_{1}$ & $\mathrm{R}_{2}$ & $\mathrm{R}_{3}$ & $\mathrm{R}_{4}$ & $\mathrm{R}_{5}$ & $\mathrm{R}_{6}$ & $\mathrm{R}_{7}$ & $\mathrm{R}_{8}$ \\
\hline 48 & $\mathrm{H}$ & $\mathrm{OH}$ & $\mathrm{H}$ & $\mathrm{OCH}_{3}$ & $\mathrm{H}$ & $\mathrm{H}$ & $\mathrm{OH}$ & $\mathrm{H}$ \\
\hline 49 & $\mathrm{H}$ & $\mathrm{OH}$ & $\mathrm{OCH}_{3}$ & $\mathrm{OCH}_{3}$ & $\mathrm{H}$ & $\mathrm{H}$ & $\mathrm{OH}$ & $\mathrm{H}$ \\
\hline 50 & $\mathrm{H}$ & $\mathrm{OCH}_{3}$ & $\mathrm{OH}$ & $\mathrm{OCH}_{3}$ & $\mathrm{H}$ & $\mathrm{H}$ & $\mathrm{OH}$ & $\mathrm{H}$ \\
\hline 51 & $\mathrm{H}$ & $\mathrm{OH}$ & $\mathrm{OCH}_{3}$ & $\mathrm{H}$ & $\mathrm{OCH}_{3}$ & $\mathrm{H}$ & $\mathrm{OH}$ & $\mathrm{H}$ \\
\hline 52 & $\mathrm{OCH}_{3}$ & $\mathrm{OH}$ & $\mathrm{H}$ & $\mathrm{H}$ & $\mathrm{OCH}_{3}$ & $\mathrm{H}$ & $\mathrm{OH}$ & $\mathrm{H}$ \\
\hline 53 & $\mathrm{H}$ & $\mathrm{OCH}_{3}$ & $\mathrm{H}$ & $\mathrm{OCH}_{3}$ & $\mathrm{H}$ & $\mathrm{H}$ & $\mathrm{OH}$ & $\mathrm{H}$ \\
\hline 54 & $\mathrm{H}$ & $\mathrm{OCH}_{3}$ & H & $\mathrm{OCH}_{3}$ & $\mathrm{H}$ & $\mathrm{H}$ & $\mathrm{OCH}_{3}$ & $\mathrm{H}$ \\
\hline 55 & $\mathrm{H}$ & $\mathrm{OCH}_{3}$ & $\mathrm{OCH}_{3}$ & $\mathrm{OCH}_{3}$ & $\mathrm{H}$ & $\mathrm{H}$ & $\mathrm{OCH}_{3}$ & $\mathrm{H}$ \\
\hline 56 & $p$-hydroxybenzyl & $\mathrm{OH}$ & $\mathrm{H}$ & $\mathrm{OCH}_{3}$ & H & $\mathrm{H}$ & $\mathrm{OH}$ & $p$-hydroxybenzyl \\
\hline 57 & $p$-hydroxybenzyl & $\mathrm{OH}$ & $\mathrm{H}$ & $\mathrm{OCH}_{3}$ & $\mathrm{H}$ & $\mathrm{H}$ & $\mathrm{OH}$ & $\mathrm{OCH}_{3}$ \\
\hline 58 & $p$-hydroxybenzyl & $\mathrm{OH}$ & $\mathrm{H}$ & $\mathrm{OCH}_{3}$ & $\mathrm{H}$ & $\mathrm{H}$ & $\mathrm{OH}$ & $\mathrm{H}$ \\
\hline 59 & $\mathrm{H}$ & $\mathrm{OH}$ & $\mathrm{H}$ & $\mathrm{OCH}_{3}$ & $\mathrm{H}$ & $\mathrm{H}$ & $\mathrm{OCH}_{3}$ & $\mathrm{H}$ \\
\hline 60 & $\mathrm{H}$ & $\mathrm{OCH}_{3}$ & $\mathrm{OH}$ & $\mathrm{OCH}_{3}$ & $\mathrm{H}$ & $\mathrm{H}$ & $\mathrm{OH}$ & $\mathrm{OCH}_{3}$ \\
\hline 61 & $\mathrm{H}$ & $\mathrm{OH}$ & $\mathrm{OCH}_{3}$ & $\mathrm{OCH}_{3}$ & $\mathrm{H}$ & $\mathrm{H}$ & $\mathrm{OH}$ & $\mathrm{H}$ \\
\hline 62 & p-hydroxybenzyl & $\mathrm{OH}$ & $\mathrm{H}$ & $\mathrm{OCH}_{3}$ & $\mathrm{H}$ & $\mathrm{H}$ & $\mathrm{OCH}_{3}$ & $\mathrm{H}$ \\
\hline 63 & $p$-hydroxybenzyl & $\mathrm{OH}$ & $\mathrm{H}$ & $\mathrm{OCH}_{3}$ & $\mathrm{H}$ & $\mathrm{H}$ & $\mathrm{OCH}_{3}$ & $\mathrm{OH}$ \\
\hline 64 & p-hydroxybenzyl & $\mathrm{OH}$ & $\mathrm{H}$ & $\mathrm{OCH}_{3}$ & $\mathrm{H}$ & $\mathrm{OH}$ & $\mathrm{OCH}_{3}$ & $\mathrm{H}$ \\
\hline 65 & p-hydroxybenzyl & $\mathrm{OH}$ & $\mathrm{OCH}_{3}$ & $\mathrm{OCH}_{3}$ & $\mathrm{H}$ & $\mathrm{H}$ & $\mathrm{OH}$ & $\mathrm{H}$ \\
\hline
\end{tabular}

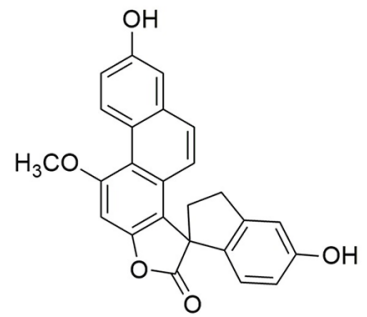

66

FIGURE 3 | Chemical structures of phenanthrenes (48-66) isolated from B. striata.

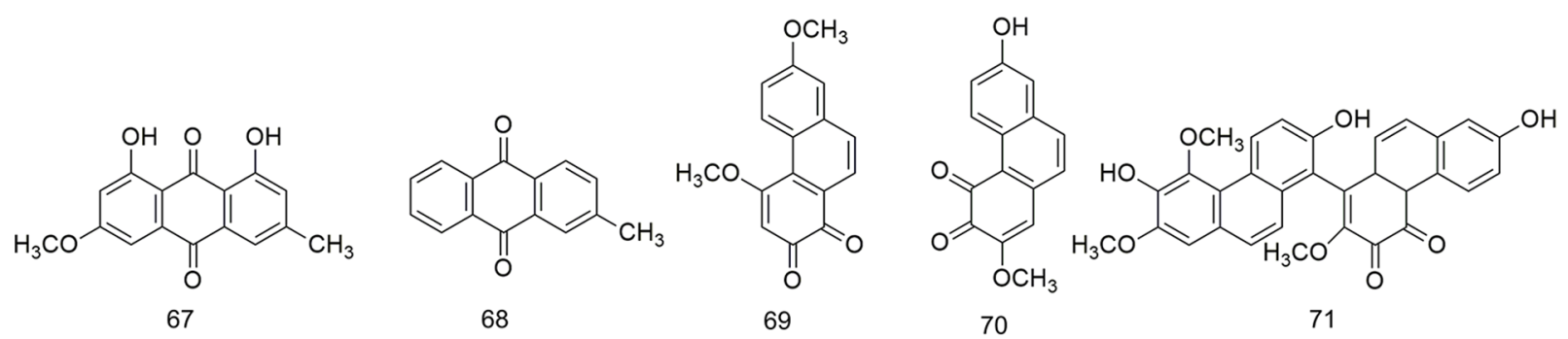

FIGURE 4 | Chemical structures of quinones (67-71) isolated from B. striata. 


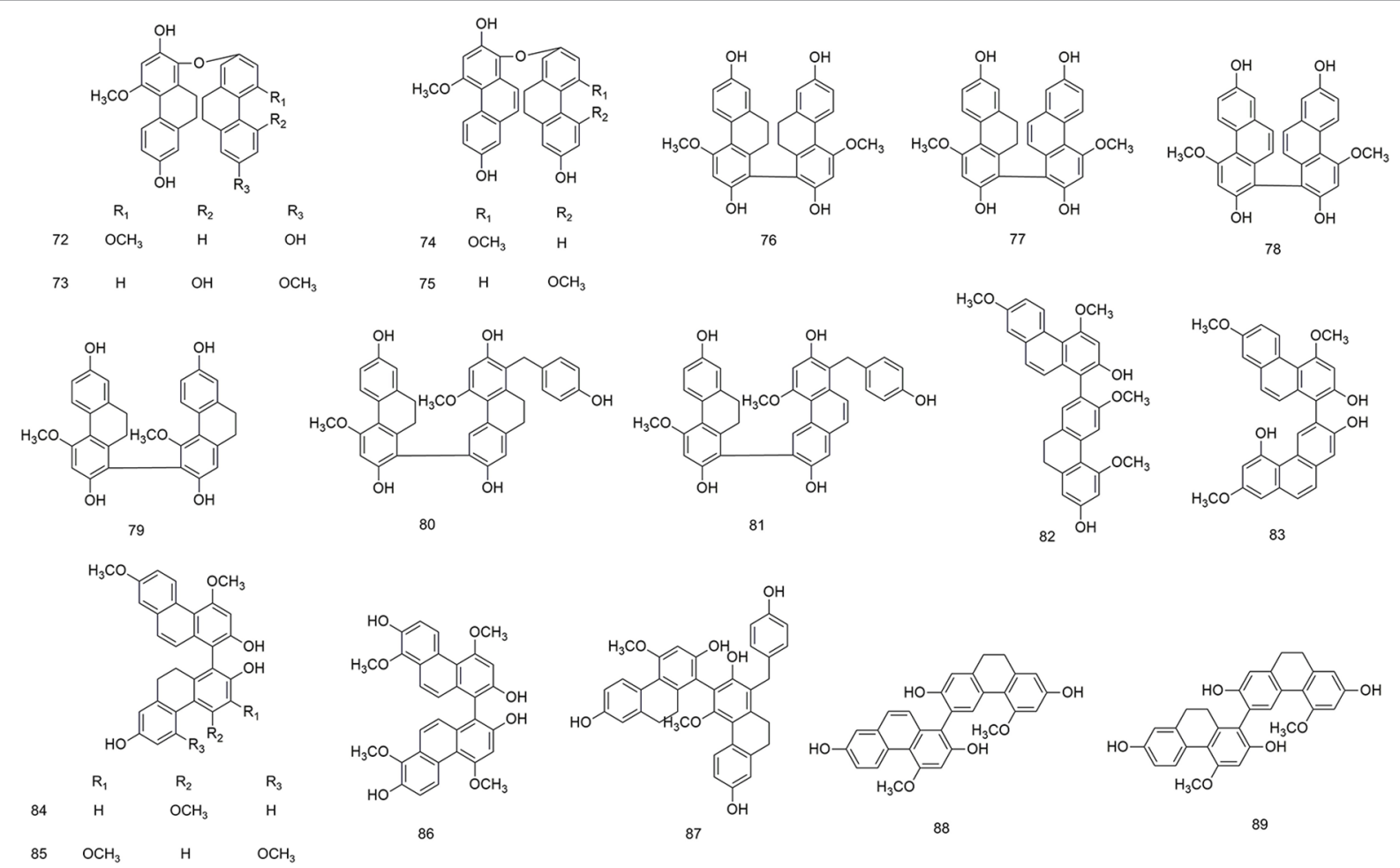

FIGURE 5 | Chemical structures of biphenanthrenes (72-89) isolated from B. striata.

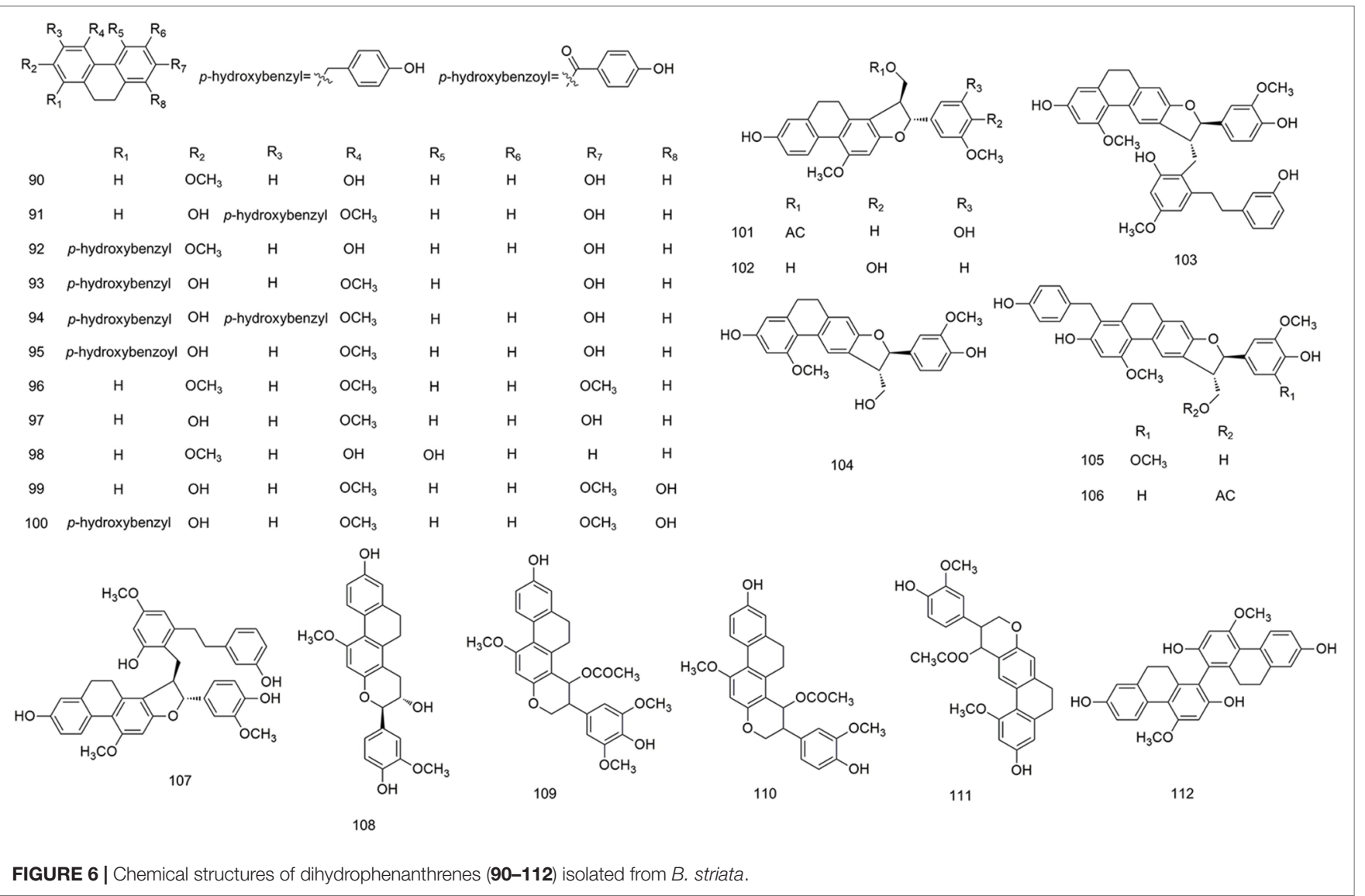




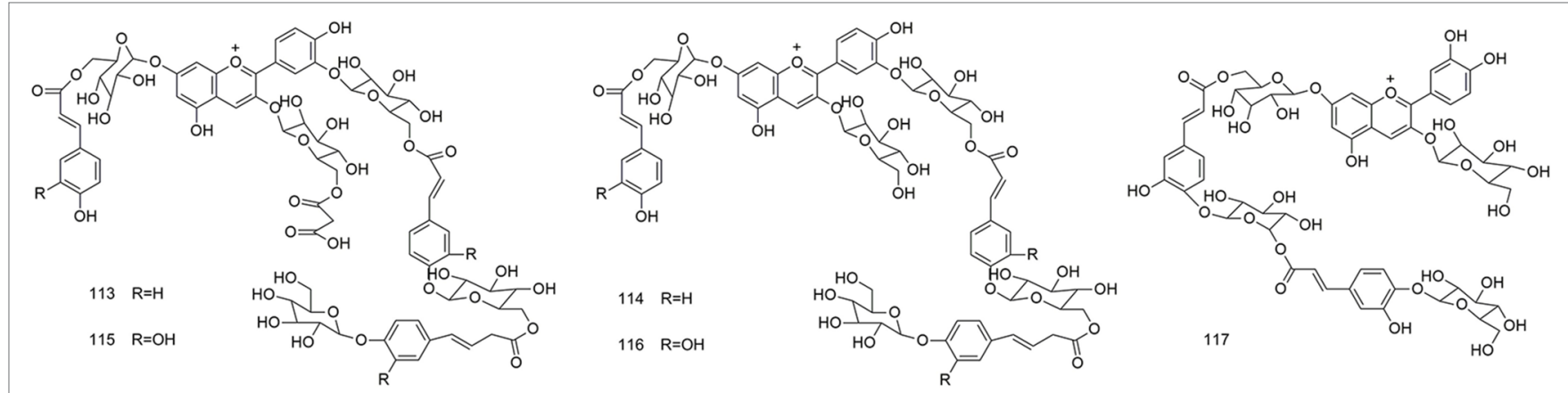

FIGURE 7 | Chemical structures of anthocyanins (113-117) isolated from B. striata.
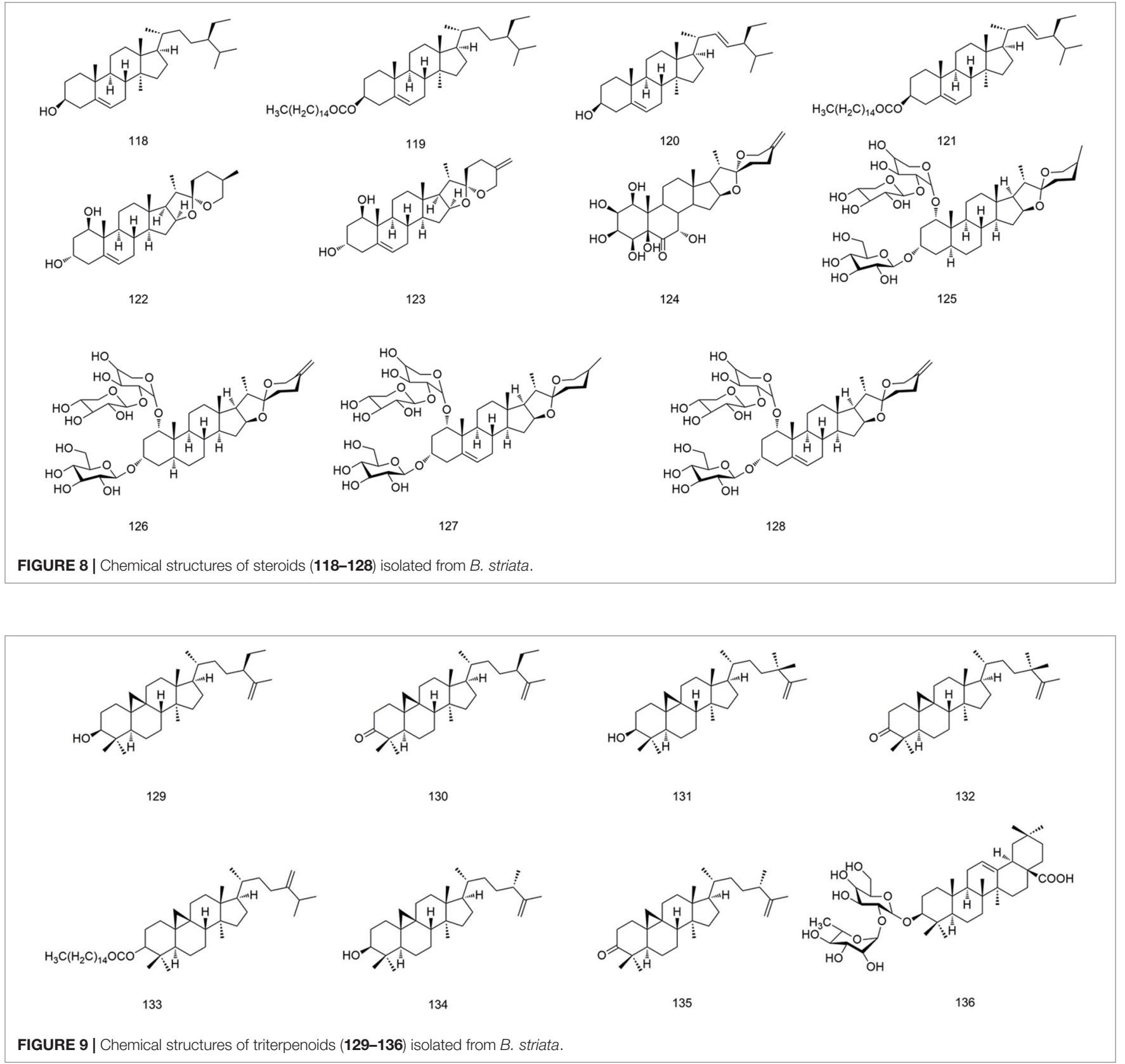

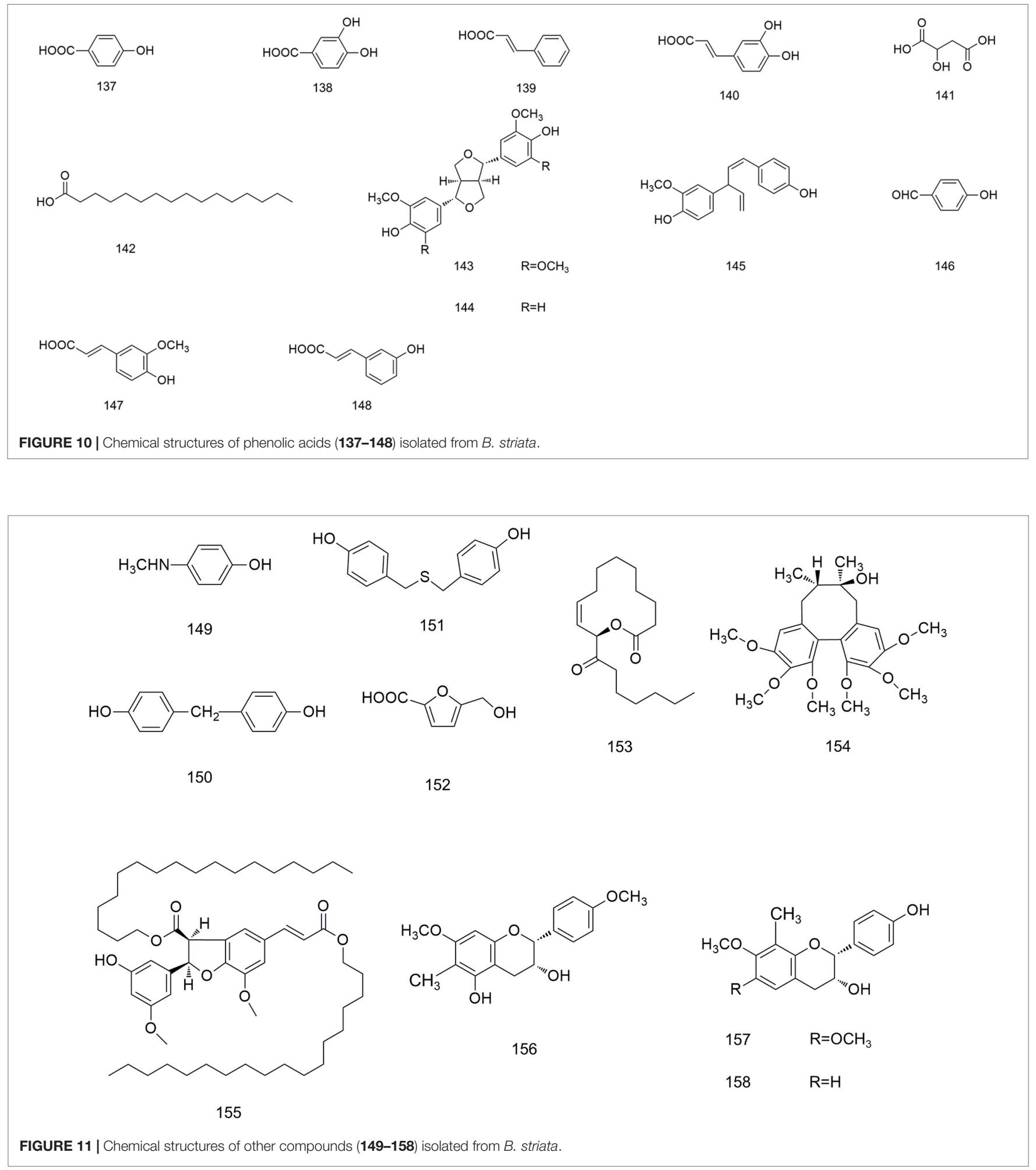

(IL)- $1 \beta$, and interferon (IFN)- $\gamma$ (Diao et al., 2008). As downstream effector molecules of the toll-like receptor-4/ lipopolysaccharide (TLR4/LPS) signaling pathway, TNF- $\alpha$ and IL- $1 \beta$ are mediators of the inflammatory response. They have similar effects and can activate various inflammatory cells (Cunha et al., 2007). Also, IFN- $\gamma$ enhances the expression of major histocompatibility complex class-II molecules on macrophage surfaces to improve their ability to present antigens (Baldridge et al., 2010). Additionally, it was found that a BSP solution (11 $\mu \mathrm{M})$ increased the nitric oxide (NO) concentration in a wound, 
which would promote the chemotaxis of neutrophils, monocytes, and macrophages, thereby providing adequate conditions for wound repair (Diao et al., 2008). NO can also regulate the diameter of and flow within blood vessels so that the wound obtains a more generous blood supply, which helps to repair the wound and advance the growth of new blood vessels. Studies have indicated that BSP can promote the cleanliness of necrotic tissue and provide conditions for subsequent tissue regeneration in a wound (Huang et al., 2019).

In the phases based on the proliferation of granulation tissue and repair, BSP can control the expression of pro-inflammatory factors (e.g., TNF- $\alpha$ ) at an appropriate level, reduce the inflammatory reaction in the wound, and prevent damage to remaining cells (Lou et al., 2010). In addition, BSP promotes an increase in the expression of the vascular endothelial growth factor (VEGF), as well as the synthesis and release of hydroxyproline. These actions promote the growth of epithelial cells, accelerate fibroblast proliferation, and further promote healing by wound contraction (Wang et al., 2006; Yu et al., 2011).

Apart from BSP, several phenolic acids (137-138, 140, 146148) in B. striata are thought to promote wound healing (Song et al., 2017). Yang and colleagues reported that the esterification products of caffeic acid (140) and phenylethyl alcohol can inhibit the transforming growth factor- $\beta 1 /$ Mothers against the decapentaplegic homolog 3 (TGF- $\beta 1 / \mathrm{Smad} 3$ ) signaling pathway (Yang et al., 2017). There is evidence that Smad3 can bind the DNA sequences of target genes at the transcriptional level, and, for pathologic skin conditions, assumes important roles in tissue repair and fibrosis (Ashcroft et al., 1999). Also, the degradation product of protocatechuic acid (138) can regulate high mobility group box (HMGB) 1 expression by modulating the HMGB1/receptor for the advanced glycation end products (RAGE) pathway (Zhang et al., 2015). During wound healing, classically activated macrophages can utilize HMGB1 to attract vessel-associated stem cells (e.g., endothelial progenitor cells, vascular progenitor cells, smooth muscle progenitor cells), which contribute to skin healing and angiogenesis (Lolmede et al., 2009).

\section{Anti-Oxidation}

Reactive oxygen species (ROS) have dual biologic effects. These are necessary to maintain cellular homeostasis during normal activities, but they also damage macromolecular matter. ROS over-accumulation results in cell death, can lead to multiple diseases, and accelerates the aging of the human body (Mittler, 2002). It is advantageous that various chemical constituents in plants can be used as natural antioxidants to remove free radicals in the body without the need for catalase, peroxidase, or superoxide dismutase (Grant and Loake, 2000).

Assays based on the scavenging of 2,2-diphenyl-1picrylhydrazyl (DPPH) radicals have shown that the fibrous root parts and pseudobulb parts of $B$. striata exhibit strong free radical-scavenging activity. Indeed, the partial antioxidant capacity of chloroform subfractions from ethanol extracts of the fibrous roots of $B$. striata was the strongest (half-maximal inhibitory concentration $\left(\mathrm{IC}_{50}\right)=0.848 \mathrm{mg} / \mathrm{L}$ ). Simultaneously, because the fibrous root parts contain more total phenols, they showed stronger reducing ability (reducing power $\mathrm{RP}_{0.5 \mathrm{AU}}=$ $83.68 \mathrm{mg} / \mathrm{L})$ (Jiang et al., 2013).

BSP has been shown to be a natural antioxidant through various antioxidant test systems (DPPH, 2,2'-azino-bis, ferricreducing antioxidant power). The antioxidative effects of BSP are mediated through the nicotinamide adenine dinucleotide phosphate oxidase $4(\mathrm{NOX} 4) / \mathrm{p} 22^{\text {phox }}$ signaling pathway (Qu et al., 2016). Through this signaling pathway, BSP can inhibit expression of NOX4 and $\mathrm{p} 22^{\text {phox }}$, thereby blocking angiotensin II-induced ROS generation (Yue et al., 2016). Furthermore, ferulic acid (147) in B. striata shows favorable free radicalscavenging ability, and its reducing ability is even stronger than that of vitamin C (Zhao et al., 2010). This antioxidant ability can protect cells from oxygen species-mediated DNA damage and radiation-induced free radicals (Srinivasan et al., 2006). The NOX4/ROS-mitogen-activated protein kinase pathway is the one through which ferulic acid (147) can decrease ethanolinduced ROS accumulation to improve anti-apoptotic responses (Li et al., 2017).

In some cases, antioxidants can worsen oxidative stress (Miller et al., 2005). If an antioxidant effect is observed, trace ROS are important cellular messengers of molecular signaling. Excessive clearance of ROS may lead to redox imbalance and induce cell-signaling disturbances, thereby triggering oxidative systems in the body.

\section{Anti-Cancer}

The anti-cytotoxic activity of synthetic drugs can strengthen some of the medicinal effects of B. striata, especially efficacy against cancer-related diseases. Glycosides (9-12), bibenzyls (30, 32-34, 38-41), phenanthrenes (57), quinines (70-71), dihydrophenanthrenes $(\mathbf{9 0}, \mathbf{9 4}, \mathbf{9 8}, 100-103,105-107,112)$, steroids (125-128), and triterpenoids (136) from B. striata have been reported to show inhibitory activities against various tumor cell lines in vitro: HepG2, MCF-7, HT-29, A549, BGC823, HL-60, MCF-7, SMMC-7721, W480, SK-OV-3, SK-MEL-2, and HCT-15. Their names and anti-cancer activities are listed in Table 2 .

Woo and colleagues revealed that compound (100) exhibited growth inhibition against a human colon cancer cell line (HCT15), with an $\mathrm{IC}_{50}$ of $2.16 \mu \mathrm{M}$ (Woo et al., 2014). Compounds (90, 98) isolated from $B$. striata tubers showed potent inhibition of proliferation of human hepatoma cells (HepG2), with an $\mathrm{IC}_{50}$ of $29.1 \mu \mathrm{M}$ and $25.5 \mu \mathrm{M}$, respectively. In HepG2 cells, these two dihydrophenanthrenes induced apoptosis by downregulating the expression of cyclin B1 and blocking the cell cycle in the G2/M phase (Wang et al., 2017). Compounds $(\mathbf{4 0 , 5 7 , 9 4 )}$ were shown to sensitize K562/breast cancer resistance protein (BCRP) cells to the metabolically active product camptothecin, SN-38, by inhibiting the function of BCRP cells, thereby demonstrating that they could alter multidrug resistance in cancer treatment (Morita et al., 2005). Compounds (39, 40) inhibited tubulin polymerization at an $\mathrm{IC}_{50}$ of $10 \mu \mathrm{M}$, and they limited cell growth by interfering with the mitosis of tumor cells (Morita et al., 2005). Compounds (70-71) exhibited significant cytotoxic effects 
TABLE 2 | Anti-cancer activities of B. striata.

\begin{tabular}{|c|c|c|c|c|}
\hline Aspects & Compound No. & Tumor Cell & $\begin{array}{l}\text { Anti-Tumor Activity } \\
\text { Mechanism }\end{array}$ & References \\
\hline \multirow[t]{8}{*}{ Directly } & 100 & HCT-15 & Inhibit growth of tumor & (Woo et al., 2014) \\
\hline & 90,98 & HepG2 & Arrest the cells at G2/M phase & (Wang et al., 2017) \\
\hline & 39,40 & K562 & Cell cycle arrest & (Morita et al., 2005) \\
\hline & 70,71 & MCF-7, HT-29, A549 & Induce apoptosis & (Sun et al., 2016a) \\
\hline & 136 & A549 & Arrest the cells at G0 phase & (Sun et al., 2016c) \\
\hline & $12,125-128$ & $\begin{array}{l}\text { A549, BGC-823, HepG2, HL-60, MCF-7, } \\
\text { SMMC-7721,W480 }\end{array}$ & Inhibit growth of tumor & (Wang and Meng, 2015) \\
\hline & $9-11$ & A549, SK-OV-3, SK-MEL-2, HCT-15 & Inhibit growth of tumor & (Wang and Meng, 2015) \\
\hline & $\begin{array}{l}30,38,40-41,101-103 \\
105-107,112\end{array}$ & HL-60, SMMC-7721, A549, MCF-7, W480 & Inhibit growth of tumor & (Li et al., 2008a) \\
\hline Indirectly & $40,59,94$ & K562 & $\begin{array}{l}\text { Reverse tumor multidrug } \\
\text { resistance }\end{array}$ & Morita et al., 2005 \\
\hline
\end{tabular}

against the MCF-7, HT-29, and A549 lines. After MCF-7 cells underwent treatment, the $\mathrm{IC}_{50}$ for compounds (70-71) was 18.49 $\mu \mathrm{g} / \mathrm{ml}$ and $12.64 \mu \mathrm{g} / \mathrm{ml}$, which are greater values than that for cisplatin. Interestingly, their anti-cancer activity is related to their pro-oxidative activity.

These compounds can block the G0/G1 phase via a ROSmediated mechanism that ultimately leads to apoptosis (Sun et al., 2016a). Compound (136) has been shown to inhibit the proliferation of tumor cells in the same manner by blocking the G0/G1 phase (Sun et al., 2016c). The $\mathrm{IC}_{50}$ for compounds (12, 125-128) against A549, BGC-823, HepG2, HL-60, MCF7, SMMC-7721, and W480 cells ranged from $11.3 \mu \mathrm{M}$ to 32.2 $\mu \mathrm{M}$, a range that is similar to that for doxorubicin (Wang and Meng, 2015). Compounds (9-11) showed significant cytotoxicity against A549, SK-OV-3, SK-MEL-2, and HCT-15 cells, with $\mathrm{IC}_{50}$ ranging from $3.98 \mu \mathrm{M}$ to $12.10 \mu \mathrm{M}$ (Wang and Meng, 2015). Compounds (30, 38, 40-41, 101-103, 105-107, 112) showed significant cytotoxicity against HL-60, SMMC-7721, A549, MCF7 , and W480 cells with $\mathrm{IC}_{50}$ ranging from $0.24 \mu \mathrm{M}$ to $38.56 \mu \mathrm{M}$ (Li et al., 2008a). Among them, compound (103) showed stronger cytotoxicity than cisplatin against the cell lines mentioned above.

In addition to the monomeric compounds stated above, BSP is also considered to have anti-cancer activity (Zhang et al., 2019b). Qian and colleagues showed that, compared with transcatheter arterial chemoembolization (TACE) alone, better results were obtained when the hepatocellular carcinoma in ACI rats was treated with a combination of TACE and arterial administration of BSP (Qian et al., 2003). BSP also inhibited the proliferation of HepG2 cells in a weak manner, and the division of HepG 2 cells was not inhibited at $<1.5 \mathrm{mg} / \mathrm{ml}$. BSP can also induce apoptosis through caspase- 3 expression (Liu et al., 2018). Using BSP as a drug carrier or mixing chemotherapeutic drugs with BSP is another anti-cancer strategy. In this manner, the drug concentration in the target organ can be maintained at a particular level. Based on this idea, copolymer micelles have been used in cancer chemotherapy (Wang et al., 2019). A macromolecular substance consisting of stearic acid-modified BSP as a carrier of docetaxel was created, and had a more pronounced effect on inhibiting the growth of HepG2 and HeLa cancer cells compared with that of docetaxel injection alone (Guan et al., 2017).

\section{Antiviral}

The efficacy of several first-line antiviral agents appears to be diminishing (Ruiz and Russell, 2012). The antiviral effect of some traditional Chinese herbs (e.g., Isatis tinctoria) has been demonstrated by experimental and clinical research ( $\mathrm{Li}$ and Peng, 2013). The active pharmaceutical ingredient in these plants can be used as the lead compound for further structural optimization to develop new antiviral drugs that can strengthen the immune system to fight and eliminate viral infections.

B. striata can exert antiviral activity by (i) interfering with the surface proteins of viruses to reduce cell infection and inhibit virus invasion into tissue; (ii) interfering with the RNA replication of the invading viruses to inhibit viral proliferation in the body; and (iii) preventing the virus from being released from host cells, thereby reducing viral spread (Shi et al., 2017; Zhang et al., 2017b). The water and ethanol extracts of $B$. striata avert invasion by the influenza virus by interfering with the hemagglutinin receptor on Madin-Darby canine kidney (MDCK) cells, and viral inhibition increases with the extract concentration, with an $\mathrm{IC}_{50}$ of $18.3 \mathrm{mg} / \mathrm{ml}$ and $235.7 \mu \mathrm{g} / \mathrm{ml}$, respectively, being recorded (Zhang et al., 2017b). Various compounds $(\mathbf{5 3}, \mathbf{8 4}-\mathbf{8 6}, \mathbf{8 8}, \mathbf{9 0}, \mathbf{9 7 - 9 8})$ in B. striata also show different levels of antiviral activity. Most of them have been shown to have significant antiviral activity against the $\mathrm{H} 3 \mathrm{~N} 2$ virus in an embryonated hen-egg model, with inhibition ranging from $17.2 \%$ to $79.3 \%$ (Shi et al., 2017).

B. striata can also assist the immune system of the human body to indirectly exert antiviral activity. Peng and colleagues investigated the immunomodulatory activity of BSPF2, which is a new polysaccharide identified from B. striata. BSPF2 significantly induced spleen cell proliferation in a dosedependent manner (Peng et al., 2014). The spleen can produce lymphocytes, macrophages, and various cytokines, and has an important role in the immune system. Animal experiments have demonstrated that B. striata can promote T-cell and B-cell immunity in immunocompromised mice (Qiu et al., 2011).

\section{Antibacterial}

The use of phytochemicals could solve the problems of drugresistant strains and antibiotic shortages. Phytochemicals are 
safe, low-toxicity agents with important bacteriostatic functions (Barbieri et al., 2017).

Testing of the antibacterial activity of $B$. striata has revealed that bibenzyls $(\mathbf{4 0}, 42)$ and biphenanthrenes (76-78, 83-86) are active against Gram-positive and Gram-negative strains. Their names and antibacterial activities are listed in Table 3. Compound (84) exhibited potential activity against Staphylococcus aureus American Type Culture Collection (ATCC) 29213, methicillin-resistant S. aureus (MRSA) ATCC 43300, and Enterococcus faecalis ATCC 29212 with a minimum

TABLE 3 | Antibacterial activities of B. striata.

\begin{tabular}{|c|c|c|c|}
\hline $\begin{array}{l}\text { Compound } \\
\text { No. }\end{array}$ & Bacteria & $\mathrm{MCl}$ & References \\
\hline \multirow[t]{3}{*}{40} & C. albicans ATCC 10257 & $>100 \mu \mathrm{g} / \mathrm{ml}$ & (Takagi et al., 1983) \\
\hline & B.cereus ATCC 11778 & $6.25 \mu \mathrm{g} / \mathrm{ml}$ & \\
\hline & S. aureus ATCC 25923 & $6.25 \mu \mathrm{g} / \mathrm{ml}$ & \\
\hline \multirow[t]{2}{*}{42} & C. albicans ATCC 10257 & $>100 \mu \mathrm{g} / \mathrm{ml}$ & (Takagi et al., 1983) \\
\hline & S. aureus ATCC 25923 & $3.12 \mu \mathrm{g} / \mathrm{ml}$ & \\
\hline \multirow[t]{3}{*}{76.77 .78} & S. aureus ATCC 25923 & $12.5-50 \mathrm{mg} / \mathrm{ml}$ & (Yang et al., 2012) \\
\hline & $\begin{array}{l}\text { S. epidermidis ATCC } \\
26069\end{array}$ & $25-50$ mg/ml & \\
\hline & B. subtilis ATCC 6633 & $25 \mathrm{mg} / \mathrm{ml}$ & \\
\hline \multirow[t]{8}{*}{78} & S. aureus ATCC 25923 & $32 \mu \mathrm{g} / \mathrm{ml}$ & (Qian et al., 2015) \\
\hline & S. aureus ATCC 29213 & $16 \mu \mathrm{g} / \mathrm{ml}$ & \\
\hline & S. aureus ATCC 43300 & $16 \mu \mathrm{g} / \mathrm{ml}$ & \\
\hline & S. epidermidis CMCC & $16 \mu \mathrm{g} / \mathrm{ml}$ & \\
\hline & 26069 & & \\
\hline & E. faecalis ATCC 29212 & $16 \mu \mathrm{g} / \mathrm{ml}$ & \\
\hline & E. coli ATCC 35218 & $>128 \mu \mathrm{g} / \mathrm{ml}$ & \\
\hline & P. vulgaris CMCC 49027 & $>128 \mu \mathrm{g} / \mathrm{ml}$ & \\
\hline \multirow[t]{8}{*}{83} & S. aureus ATCC 25923 & $8 \mu \mathrm{g} / \mathrm{ml}$ & (Qian et al., 2015) \\
\hline & S. aureus ATCC 29213 & $8 \mu \mathrm{g} / \mathrm{ml}$ & \\
\hline & S. aureus ATCC 43300 & $8 \mu \mathrm{g} / \mathrm{ml}$ & \\
\hline & S. epidermidis CMCC & $8 \mu \mathrm{g} / \mathrm{ml}$ & \\
\hline & 26069 & & \\
\hline & E. faecalis ATCC 29212 & $64 \mu \mathrm{g} / \mathrm{ml}$ & \\
\hline & E. coli ATCC 35218 & $>128 \mu \mathrm{g} / \mathrm{ml}$ & \\
\hline & P. vulgaris CMCC 49027 & $>128 \mu \mathrm{g} / \mathrm{ml}$ & \\
\hline \multirow[t]{8}{*}{84} & S. aureus ATCC 25923 & $4 \mu \mathrm{g} / \mathrm{ml}$ & (Qian et al., 2015) \\
\hline & S. aureus ATCC 29213 & $2 \mu \mathrm{g} / \mathrm{ml}$ & \\
\hline & S. aureus ATCC 43300 & $4 \mu \mathrm{g} / \mathrm{ml}$ & \\
\hline & S. epidermidis CMCC & $4 \mu \mathrm{g} / \mathrm{ml}$ & \\
\hline & 26069 & & \\
\hline & E. faecalis ATCC 29212 & $4 \mu \mathrm{g} / \mathrm{ml}$ & \\
\hline & E. coli ATCC 35218 & $>128 \mu \mathrm{g} / \mathrm{ml}$ & \\
\hline & P. vulgaris CMCC 49027 & $>128 \mu \mathrm{g} / \mathrm{ml}$ & \\
\hline \multirow[t]{8}{*}{85} & S. aureus ATCC 25923 & $64 \mu \mathrm{g} / \mathrm{ml}$ & (Qian et al., 2015) \\
\hline & S. aureus ATCC 29213 & $32 \mu \mathrm{g} / \mathrm{ml}$ & \\
\hline & S. aureus ATCC 43300 & $32 \mu \mathrm{g} / \mathrm{ml}$ & \\
\hline & S. epidermidis CMCC & $8 \mu \mathrm{g} / \mathrm{ml}$ & \\
\hline & 26069 & & \\
\hline & E. faecalis ATCC 29212 & $>128 \mu \mathrm{g} / \mathrm{ml}$ & \\
\hline & E. coli ATCC 35218 & $>128 \mu \mathrm{g} / \mathrm{ml}$ & \\
\hline & P. vulgaris CMCC 49027 & $>128 \mu \mathrm{g} / \mathrm{ml}$ & \\
\hline \multirow[t]{8}{*}{86} & S. aureus ATCC 25923 & $16 \mu \mathrm{g} / \mathrm{ml}$ & (Qian et al., 2015) \\
\hline & S. aureus ATCC 29213 & $8 \mu \mathrm{g} / \mathrm{ml}$ & \\
\hline & S. aureus ATCC 43300 & $16 \mu \mathrm{g} / \mathrm{ml}$ & \\
\hline & S. epidermidis CMCC & $8 \mu \mathrm{g} / \mathrm{ml}$ & \\
\hline & 26069 & & \\
\hline & E. faecalis ATCC 29212 & $64 \mu \mathrm{g} / \mathrm{ml}$ & \\
\hline & E. coli ATCC 35218 & $>128 \mu \mathrm{g} / \mathrm{ml}$ & \\
\hline & P. vulgaris CMCC 49027 & $>128 \mu \mathrm{g} / \mathrm{ml}$ & \\
\hline
\end{tabular}

inhibitory concentration (MIC) of $2-8 \mu \mathrm{g} / \mathrm{ml}$. Scanning electron microscopy showed that compound (84) killed bacterial cells by damaging their cytoplasmic membranes (Chen et al., 2018). Compounds of 78 and 83-86, with a MIC of $2 \mu \mathrm{g} / \mathrm{ml}$ and 64 $\mu \mathrm{g} / \mathrm{ml}$, were active against six Gram-positive bacteria: $S$. aureus ATCC 25923, 29213, 43300, Staphylococcus epidermidis Center for Medical Culture Collections (CMCC) 26069, E. faecalis ATCC 29212, and Bacillus subtilis China General Microbiological Culture Collection Center (CGMCC) 1.1470 (Qian et al., 2015). Blestriarene A (76), blestriarene B (77), and blestriarene C (78) showed activity against $S$. aureus ATCC 25923 at MIC of $12.5-50 \mathrm{mg} / \mathrm{ml}$, against S. epidermidis ATCC 26069 at MIC of $25-50 \mathrm{mg} / \mathrm{ml}$, and against B. subtilis ATCC 6633 at MIC of 25 $\mathrm{mg} / \mathrm{ml}$ (Yang et al., 2012). Compound (40) exhibited in vitro activity against Bacillus cereus ATCC 11778 and S. aureus ATCC 25923 , with a MIC of $6.25 \mu \mathrm{g} / \mathrm{ml}$, and the MIC for compound (42) against $S$. aureus ATCC 25923 was $3.12 \mu \mathrm{g} / \mathrm{ml}$ (Takagi et al., 1983). Furthermore, the phenanthrene fraction from the ethanol extract of $B$. striata has been regarded as a significantly active agent against Gram-positive bacteria, including clinical isolates of MRSA and S. aureus (S. aureus ATCC 25923, 29213, 43300) (Guo et al., 2016).

\section{CLINICAL APPLICATION}

\section{TCM Formulations}

Based on classical theories of TCM, Chinese medicinal formulations are prepared by pooling different types of medicinal herbs together (Li and Peng, 2013). During the development of TCM theory, many formulations have been recorded in pharmacopeias or by folklore based on clinical experiences. Considering the different causes and symptoms of diseases, $B$. striata is often used with other drugs to offset the toxicity of one drug or enhance the bioavailability of another drug, which is known as the "correspondence of prescription and syndrome" in TCM.

For example, Bai Ji San (B. striata liniment) is frequently used as an astringent hemostatic medicine. It is composed of $B$. striata (Bai Ji in Chinese), Asarum sieboldii (Xi Xin), Saposhnikovia divaricata (Fang Feng), and Semen Platycladi (Bai Zi Ren), as noted in Tai Ping Sheng Hui Fang (medical literature edited by the Song Dynasty government). Qu Huo Wai Xiao Tang (decoction for purging fire) can be used to treat skin scalding. It is composed of Bai Ji, Sanguisorba officinalis (Di Yu), Cacumen Platycladi (Bai Ye), stir-baked Fructus Gardeniae (Chao Zhi Zi), Cynanchum otophyllum (Qing Yang Sheng), Angelica sinensis (Dang Gui), and Radix Glycyrrhizae (Gan Cao), as noted in Dong Tian Ao Zhi (Practical Surgical and Clinical Experiences). Although the pharmacologic mechanism of these formulations is not clear, considerable clinical evidence suggests that $B$. striata has value in the treatment of various diseases.

\section{Embolizing Agent}

TACE is a first-line treatment for most inoperable tumors (Tsurusaki and Murakami, 2015). An embolizing agent based 
on B. striata and TACE has been used in clinical applications. The B. striata embolizing agent can, in general, be classified into three types according to its formulation: liquid, compound, or microspheres.

The B. striata liquid embolizing agent is composed of BSP, cellulose diacetate (solute), and dimethyl sulfoxide (solvent). It has the characteristics of easy flow, good biocompatibility, and no fixed morphology. It can be used for the embolization of irregular tumor cavities and reduces damage to vascular walls (Sun et al., 2005).

The B. striata compound embolizing agent is a synthetic moiety that can be used as an anti-carcinogen and coagulant. In this compound, B. striata can have a pharmacologic role with other medicinal ingredients. It can inhibit the proliferation and spread of tumor cells, and reduce the risk of adverse reactions (Chen et al., 2006).

B. striata polysaccharide microspheres (BSPMs) could be promising transarterial chemoembolization carriers for cancer treatment. BSPMs show favorable drug-loading, swelling, suspension, drug-entrapment, and release characteristics in vitro, which are conducive to long-term targeted chemotherapy (Li et al., 2018b). Studies have shown that BSPMs can embolize the blood supply of hepatic arteries and the hepatic portal vein, and completely inhibit the growth of tumors and surrounding microvessels by stopping the binding of VEGF to its receptor (Zhao et al., 2004).

The B. striata embolizing agent has also performed well in TACE of hepatic cirrhosis with portal hypertension and secondary hypersplenism. In a study by Liu and coworkers, long-term follow-up of surgical patients revealed a mean survival duration of $61.5 \pm 9.1$ (median, 60; range, 1-157) months in the control group and $63.4 \pm 9.9(52,0-161)$ months in the $B$. striata group. The spleen thickness of treated patients was reduced compared with that before TACE, and the counts of white blood cells, platelets, and red blood cells returned to within normal ranges (Liu et al., 2011). Therefore, TACE using $B$. striata as an embolizing agent is a safe and efficacious treatment for patients with hepatic cirrhosis with portal hypertension and secondary hypersplenism.

\section{Mucosa-Protective Agent}

The imbalance between proteases and mucosal defense factors is an important cause of several digestive-system diseases (e.g., gastric ulcers, duodenal ulcers, and ulcerative colitis). In recent years, strengthening mucosal defenses has become a new strategy to treat peptic ulcers (Al-Jiboury and Kaunitz, 2012). B. striata can be used as a mucosa-protective agent alone or in combination with other Chinese herbal medicines (Zhang et al., 2019a). It can be administered by gastroscopic spraying, injection through a gastric tube, oral administration, or enemas (Zhou et al., 2019).

In the treatment of diseases of the upper gastrointestinal tract, $B$. striata forms a protective film on the damaged mucosal surface to protect it from erosion (by gastric acid) and digestion (by pepsin), thus enhancing the defensive function of the gastric mucosa (He et al., 2016). A preliminary clinical trial showed that
B. striata combined with Panax notoginseng, omeprazole, and amoxicillin could reduce the inflammatory response of gastric ulcers, protect the ulcer surface, and promote the regeneration and repair of ulcerated tissue (Bai and Zhang, 2010). In the treatment of ulcerative colitis, $B$. striata can be added to an enema to act as a mucosa-protective agent. After $B$. striata powder enters the intestine, it can form a gel on the surface of the intestinal mucosa to protect it. It can also enhance adhesion of drugs to the intestinal wall, thereby increasing the drug concentration in the intestinal tract.

B. striata has a hemostatic function and can promote ulcer healing, which can aid regeneration of the intestinal mucosa and, thus, promote the healing of ulcerative colitis (Lan and Chang, 2014). BSP can alleviate the pathologic damage and symptoms of ulcerative colitis. It can inhibit the expression of TNF- $\alpha$ and nuclear factor-kappa B, upregulate the expression of IL-10, prevent the abnormal release of proinflammatory factors, promote repair of the intestinal mucosa, and inhibit inflammation (Ke and Zhao, 2011). BSP can also reduce the expression of Th2 cytokines in the colon by inhibiting macrophage activation, and relieve inflammation in the intestinal tract. Furthermore, enemas containing B. striata can reduce the risk of postoperative complications of patients with an artificial anus in the colon, reduce the possibility of inflammatory reactions in the surrounding skin, reduce erosion and hemorrhage in the artificial anal mucosa, and improve the quality of life for patients (Lan and Chang, 2014).

\section{Novel Biomaterials}

Recently, novel "dissolving microneedles" have been created using BSP. Microneedles can carry drugs into the skin for drug delivery and release encapsulated drugs over time. Compared with conventional transdermal patches and subcutaneous injections, B. striata polysaccharide microneedles are a minimally invasive method of drug delivery that prevents skin retention of biohazardous sharp waste. The pharmacologic activity of BSP contributes to the recovery from the micro-trauma caused by microneedles, such as prevention of bacterial infection and reduction of inflammation (Hu et al., 2018).

Mixing B. striata and polyvinyl alcohol to form a dressing matrix can result in a novel, high-quality biologic dressing. This material makes full use of the pharmacologic activity of $B$. striata for hemostasis and wound healing, and has been shown to have good mechanical properties and biocompatibility. Hence, it can absorb oozing blood and tissue fluid, which accelerates the healing of burns, surgical wounds, and acute wounds (Lin et al., 2012).

A novel, readily stripped bilayer composite was designed as a wound-dressing material, and exhibited excellent biocompatibility and mechanical properties. This composite comprised an upper layer of soybean protein nonwoven fabric coated with a lower layer of genipin-crosslinked chitosan and $B$. striata herbal extract. The extract in the wound-dressing material was non-toxic, but also promoted the growth of L929 fibroblasts, which are beneficial for wound healing (Liu and Huang, 2010). 


\section{Quality Control}

The China Food and Drug Administration defines "geoauthentic" herbs as traditional Chinese crude drugs grown in unadulterated environments and subjected to natural conditions, or with specific cultivation techniques and processing methods (Brinckmann, 2013). Zhengan County in Guizhou Province is accepted as the most optimal location to produce the crude tubers of B. striata in China. The B. striata produced here is called "Zheng'an Bai Ji". However, the material basis and potential mechanisms for producing geo-authentic herbs are not completely clear. The Chinese Pharmacopoeia recommends identifying the geo-genuine properties of $B$. striata according to morphologic, microscopic, and thin-layer chromatography approaches, and by ensuring that the residual inorganic components after ashing are $\leq 15.0 \%$ (He et al., 2016).

The standards mentioned above are accepted in formularies and pharmacopoeias, but may not be sufficient to evaluate the quality of all B. striata tubers. The latter contain various medicinally active ingredients (e.g., glycosides, bibenzyls, phenanthrenes, biphenanthrenes, and dihydrophenanthrenes) which should also be considered in quality control. For example, assessment of amounts of dactylorhin A (1), gymnoside V (5), gymnoside IX (6), militarine (8), and bletilnoside A (9) by ultra-high-performance liquid chromatography using photo-diode array detection showed obvious variation in response to the different origins of $B$. striata. The mass fraction of each chemical marker was $0.341-1.110,2.840-6.990$, $5.790-34.400,0.191-3.890$, and $0.184-5.050 \mathrm{mg} / \mathrm{g}$, respectively. Among them, a wild strain of $B$. striata produced in Zhengan County contained relatively high levels of active ingredients at $1.110,6.500,31.400,3.890$, and $5.05 \mathrm{mg} / \mathrm{g}$, respectively (Wang et al., 2014). Furthermore, Liu et al. used a parallel-line assay based on quantitative responses to evaluate the hemostatic potency of $B$. striata obtained from different habitats, which provided monitoring indicators for the clinical efficacy of B. striata (Liu et al., 2014). These preliminary results may provide evidence for the geographic specificity and quality control of geo-authentic herbs.

\section{Toxicology}

Toxic side effects and adverse reactions from $B$. striata have rarely been reported. If $B$. striata is used with Aconitum carmichaeli, it enhances the content of hypaconitine in the decoction (Weng et al., 2004). However, hypaconitine can increase the expression of $\mathrm{RyR}_{2}$ (a regulatory protein related to the function of calcium channels) in cardiac muscle, which may result in an abnormal heart rate (Liu et al., 2016). Therefore, B. striata cannot be used with A. carmichaeli in TCM (He et al., 2016).

Tests to determine the acute toxicity of $B$. striata have shown that the mean mortality of mice is $<20 \%$ if a single intragastric dose is increased to $80 \mathrm{~g} / \mathrm{kg}$ body weight, and the median lethal dose of $B$. striata was not detected. Subsequent experiments using a maximum dose of $B$. striata that did not cause the death of experimental animals was determined to be $180 \mathrm{~g} / \mathrm{kg}$ body weight (Zhang et al., 2013).

A series of toxicology experiments showed that BSP did not elicit allergic reactions, phototoxicity reactions and, most importantly, no obvious adverse reactions to human skin. These experiments included tests of acute oral toxicity (in mice), skin stimulation (rabbits), skin allergy (guinea pigs), skin phototoxicity (guinea pigs), and skin patches (humans) (Zhang et al., 2003). Yue and colleagues showed that the acute toxicity of BSP was very low. Mice were given BSP ( $4 \mathrm{~g} / \mathrm{kg}$ body weight, i.g.) twice during an interval of $6 \mathrm{~h}$. None of the mice died, and no significant changes were detected in their activity, food intake, fur, or weight (Yue et al., 2003).

\section{CONCLUSIONS AND PERSPECTIVES}

B. striata has been used as a medicinal herb in China for thousands of years. However, due to the high degree of personalization of the diagnosis and treatment of TCM, its clinical efficacy cannot be comprehensively evaluated by evidence-based medicine. In this review, we categorized research on B. striata based on its chemical constituents, pharmacologic activities, and clinical applications. We also tried to establish connections between the conclusions of many studies carried out on B. striata.

More than 150 compounds have been isolated from various parts of $B$. striata, and they exhibit a wide range of biologic and pharmacologic properties. Efficacious use of B. striata is dependent upon the connection between these chemical components and their specific bioactivities. Based on extensive biologic testing, numerous phytochemicals identified in B. striata are efficacious against one or more diseases. Developing and applying monomer compounds isolated from this herb is another development direction. Therefore, B. striata has great potential to be further mined for its pharmacological effects.

These studies on component detection, pharmacological probing, and clinical exploration conducted on B. striata have provided us with additional data for this valuable herb. Additional systematic studies will offer sufficient proof for establishing the efficacy and safety of this herb so it can be used as a medicine from a scientific point of view. We hope that this review will allow further research and development of this unique plant.

\section{AUTHOR CONTRIBUTIONS}

Conceptualization: DX. Project administration: DX, JC. Writing original draft: YP. Writing - review and editing: DX, JC.

\section{FUNDING}

This research was financially supported by the National Natural Science Foundation of China (31560079, 31560102, 31960074), the PhD Science Foundation of Zunyi Medical University (F-809), the Talent Growth Project of the Guizhou Education Department (KY[2017]194), and the Research Project of Guizhou Administration of Traditional Chinese Medicine (QZYY-2019-060).

\section{ACKNOWLEDGMENTS}

We are grateful to Dr. Surendra Sarsaiya (ZMU, China) for proofreading an earlier version of this article. 


\section{REFERENCES}

Al-Jiboury, H., and Kaunitz, J. D. (2012). Gastroduodenal mucosal defense. Curr. Opin. Gastroen. 28, 594-601. doi: 10.1097/00001574-199012000-00003

Ashcroft, G. S., Yang, X., Glick, A. B., Weinstein, M., Letterio, J. J., Mizel, D. E., et al. (1999). Mice lacking Smad3 show accelerated wound healing and an impaired local inflammatory response. Nat. Cell Biol. 1, 260-266. doi: 10.1038/12971

Bae, J. Y., Lee, J. W., Jin, Q., Jang, H., Lee, D., Kim, Y., et al. (2016). Chemical constituents isolated from Bletilla striata and their inhibitory effects on nitric oxide production in RAW 264.7 Cells. Chem. Biodivers. 14, e1600243. doi: 10.1002/cbdv.201600243

Bai, L., Kato, T., Inoue, K., Yamaki, M., and Takagi, S. (1991). Blestrianol A, B and C, biphenanthrenes from Bletilla striata. Phytochemistry 30, 2733-2735. doi: 10.1016/0031-9422(91)85133-K

Bai, L., Kato, T., Inoue, K., Yamaki, M., and Takagi, S. (1993). Stilbenoids from Bletilla striata. Phytochemistry 33, 1481-1483. doi: 10.1016/0031-9422(93)85115-8

Bai, L., Yamaki, M., Inoue, K., and Takagi, S. (1990). Blestrin A and B, bis(dihydrophenanthrene)ethers from Bletilla striata. Phytochemistry 29, 1259-1260. doi: 10.1016/0031-9422(90)85437-K

Bai, X. M., and Zhang, Q. Y. (2010). Observation of baijigou powder, notoginseng powder and cuttlefish bone jointed with omeprazole and amoxicillin in the treatment of gastric ulcer. Chin. Med. Herald 7, 79-80. doi: 10.3969/j. issn.1673-7210.2010.03.044

Baldridge, M. T., King, K. Y., Boles, N. C., Weksberg, D. C., and Goodell, M. A. (2010). Quiescent haematopoietic stem cells are activated by IFN-gamma in response to chronic infection. Nature 465, 793-797. doi: 10.1038/nature09135

Barbieri, R., Coppo, E., Marchese, A., Daglia, M., Sobarzo-Sánchez, E., Nabavi, S. F., et al. (2017). Phytochemicals for human disease: An update on plant-derived compounds antibacterial activity. Microbiol. Res. 196, 44-68. doi: 10.1016/j.micres.2016.12.003

Brinckmann, J. A. (2013). Emerging importance of geographical indications and designations of origin-authenticating geo-authentic botanicals and implications for phytotherapy. Phytother. Res. 27, 1581-1587. doi: 10.1002/ ptr.4912

Chen, B. C., Lin, C. X., Chen, N. P., Gao, C. X., Zhao, Y. J., and Qian, C. D. (2018). Phenanthrene antibiotic targets bacterial membranes and kills Staphylococcus aureus with a low propensity for resistance development. Front. Microbiol. 9, 1593-1602. doi: 10.3389/fmicb.2018.01593

Chen, J., Lv, L. Y., Li, Y., Ren, X. D., Luo, H., Gao, Y. P., et al. (2019). Preparation and evaluation of Bletilla striata polysaccharide/graphene oxide composite hemostatic sponge. Int. J. Biol. Macromol. 130, 827-835. doi: 10.1016/j. ijbiomac.2019.02.137

Chen, Z. Q., Yang, X. Z., Shen, J. J., Wang, S. D., and Zhen, X. G. (2006). The experimental studies of Chinese herbs as a vascular embolization agent for the hepatic arteries. J. Interventronal. Radiol. 15, 88-92. doi: 10.3969/j. issn.1008-794X.2006.02.009

Chen, Z. Y., Cheng, L. Z., He, Y. C., and Wei, X. L. (2018). Extraction, characterization, utilization as wound dressing and drug delivery of Bletilla striata polysaccharide: a review. Int. J. Biol. Macromol. 120, 2076-2085. doi: 10.1016/j.ijbiomac.2018.09.028

Cunha, T. M., Verri, W. A., Fukada, S. Y., Guerrero, A. T. G., SantodomingoGarzón, T., Poole, S., et al. (2007). TNF- $\alpha$ and IL-1 $\beta$ mediate inflammatory hypernociception in mice triggered by $\mathrm{B}_{1}$ but not $\mathrm{B}_{2}$ kinin receptor. Eur. J. Pharmacol. 573, 221-229. doi: 10.1016/j.ejphar.2007.07.007

Dai, Y., and Sun, L. R. (2008). Advances in studies on bibenzyls naturally occurred in plant. Chin. Tradit. Herbal. Drugs 39, 1753-1755. doi: 10.3321/j. issn:0253-2670.2008.11.051

Debieu, D., Gall, C., Gredt, M., Bach, J., Malosse, C., and Leroux, P. (1992). Ergosterol biosynthesis and its inhibition by fenpropimorph in Fusarium species. Phytochemistry 31, 1223-1233. doi: 10.1016/0031-9422(92)80265-G

Diao, H. J., Li, X., Chen, J. N., Luo, Y., Chen, X., Dong, L., et al. (2008). Bletilla striata polysaccharide stimulates inducible nitric oxide synthase and proinflammatory cytokine expression in macrophages. J. Biosci. Bioeng. 105, 85-89. doi: 10.1263/ jbb. 105.85

Dong, L., Dong, Y. X., Liu, X. X., Liao, S. G., Wang, A. M., and Li, Y. J. (2014). Effect of Bletilla striata polysaccharides on rat platelet aggregation, coagulation function, $\mathrm{TXB}_{2}$ and 6-keto-PGF ${ }_{1 a}$ expression. J. Guiyang Med. Coll. 39, 459462. doi: 10.19367/j.cnki.1000-2707.2014.04.004
Feng, J. Q., Zhang, R. J., and Zhao, W. M. (2008). Novel bibenzyl derivatives from the tubers of Bletilla striata. Helv. Chim. Acta 91, 520-525. doi: 10.1002/ hlca. 200890056

Gachet, C. (2006). Regulation of platelet functions by P2 receptors. Annu. Rev. Pharmacol. 46, 277-300. doi: 10.1146/annurev.pharmtox.46.120604.141207

Grant, J. J., and Loake, G. J. (2000). Role of reactive oxygen intermediates and cognate redox signaling in disease resistance. Plant Physiol. 124, 21-29. doi: 10.1104/pp.124.1.21

Gu, R. H., Hong, L. Y., and Long, C. L. (2013). The ways of producing secondary metabolites via plant cell culture. Plant Physiol. 9, 869-881. doi: 10.13592/j. cnki.ppj.2013.09.016

Guan, Q. X., Zhang, G. Y., Sun, D. D., Wang, Y., Liu, K., Wang, M., et al. (2017). In vitro and in vivo evaluation of docetaxel-loaded stearic acid-modified Bletilla striata polysaccharide copolymer micelles. PLos One 12, e0173172. doi: 10.1371/journal.pone.0173172

Guo, J. J., Dai, B. L., Chen, N. P., Jin, L. X., Jiang, F. S., Ding, Z. S., et al. (2016). The anti-Staphylococcus aureus activity of the phenanthrene fraction from fibrous roots of Bletilla striata. BMC Complem. Altern. M. 16, 491-497. doi: 10.1186/ s12906-016-1488-Z

Han, G. X., Wang, L. X., Wang, M. L., Zhang, W. D., Li, T. Z., Liu, W. Y., et al. (2001). Studies on the chemical constituents of Bletilla striata. J. Pharm. Pract. 19, 360-361. doi: 10.3969/j.issn.1006-0111.2001.06.018

Han, G. X., Wang, L. X., Zhang, W. D., Wang, M. L., Liu, H. T., and Xiao, J. H. (2002a). Studies on chemical constituents of Bletilla striata (II). Acad. J. Second Mil. Med. Univ. 23, 1029-1031. doi: 10.16781/j.0258-879x.2002.09.036

Han, G. X., Wang, L. X., Zhang, W. D., Yang, Z., Li, T. Z., Jiang, T., et al. (2002b). Study on chemical constituents of Bletilla striata(I). Acad. J. Second Mil. Med. Univ. 23, 443-445. doi: 10.16781/j.0258-879x.2002.04.038

Han, G. X., Wang, L. X., Zheng Bing, G. U., Zhang, W. D., and Chen, H. S. (2002c). A new bibenzyl derivative from Bletilla striata. Chin. Chem. Lett. 13, 231-232. doi: $10.1021 / \mathrm{cm} 022001 \mathrm{r}$

He, X. R., Wang, X. X., Fang, J. C., Zhao, Z. F., Huang, L. H., Hao, G., et al. (2016) Bletilla striata: Medicinal uses, phytochemistry and pharmacological activities. J. Ethnopharmacol. 195, 20-38. doi: 10.1016/j.jep.2016.11.026

Heleno, S. A., Martins, A., Queiroz, M. J. R. P., and Ferreira, I. C. F. R. (2015). Bioactivity of phenolic acids: metabolites versus parent compounds: a review. Food Chem. 173, 501-513. doi: 10.1016/j.foodchem.2014.10.057

Hossain, M. M. (2011). Therapeutic orchids: traditional uses and recent advances-an overview. Fitoterapia 82, 102-140. doi: 10.1016/j.fitote.2010.09.007

Hu, L. L., Liao, Z. C., Hu, Q. Q., Maffucci, K. G., and Qu, Y. (2018). Novel Bletilla striata polysaccharide microneedles: fabrication, characterization, and in vitro transcutaneous drug delivery. Int. J. Biol. Macromol. 117, 928-936. doi: 10.1016/j.ijbiomac.2018.05.097

Huang, Y., Shi, F., Wang, L., Yang, Y., Khan, B. M., Cheong, K. L., et al. (2019). Preparation and evaluation of Bletilla striata polysaccharide/carboxymethyl chitosan/Carbomer 940 hydrogel for wound healing. Int. J. Biol. Macromol. 132, 729-737. doi: 10.1016/j.ijbiomac.2019.03.157

Hung, H. Y., and Wu, T. S. (2016). Recent progress on the traditional Chinese medicines that regulate the blood. J. Food Drug Anal. 24, 221-238. doi: 10.1016/j.jfda.2015.10.009

Jiang, F. S., Li, W. P., Huang, Y. F., Chen, Y. T., Jin, B., Chen, N. P., et al. (2013). Antioxidant, antityrosinase and antitumor activity comparison: the potential utilization of fibrous root part of Bletilla striata (Thunb.) Reichb.f. PLos One 8, e58004. doi: 10.1371/journal.pone.0058004

Kasuya, A., and Tokura, Y. (2014). Attempts to accelerate wound healing. J. Dermatol. Sci. 76, 169-172. doi: 10.1016/j.jdermsci.2014.11.001

Ke, C. Y., and Zhao, C. J. (2011). Effects of Bletilla striata polysaccharides on ulcerative colitis. Chin. Pharm. 22, 2132-2134. doi: 10.1007/s10008-010-1224-4

Lan, H., and Chang, M. Q. (2014). Application of Bletilla striata in diagnosis and treatment of anorectal diseases. Chin. J. Coloproctol. 34, 53-55. doi: 10.3969/j. issn.1000-1174.2014.12.027

Li, C., Li, L., Yang, C. F., Zhong, Y. J., Wu, D., Shi, L., et al. (2017). Hepatoprotective effects of Methyl ferulic acid on alcohol-induced liver oxidative injury in mice by inhibiting the NOX4/ROS-MAPK pathway. Biochem. Bioph. Res. Co. 493, 277-285. doi: 10.1016/j.bbrc.2017.09.030

Li, J. Y., Kuang, M. T., Yang, L., Kong, Q. H., Hou, B., Liu, Z. H., et al. (2008a). Stilbenes with anti-inflammatory and cytotoxic activity from the rhizomes of Bletilla ochracea Schltr. Fitoterapia 127, 47-80. doi: 10.1016/j.fitote.2018.02.007 
Li, T., and Peng, T. (2013). Traditional Chinese herbal medicine as a source of molecules with antiviral activity. Antiviral. Res. 97, 1-9. doi: 10.1016/j. antiviral.2012.10.006

Li, W.Z., Zhao, N., Chen, Z., Han, W.X., Fu, L. N., He, S. M., et al. (2018b). Preparation of matrine loaded microspheres based on Bletilla striata polysaccharide. Acta Pharmacol. Sin. 53, 284-290. doi: 10.16438/j.0513-4870.2017-0903

Lin, J. H., Lu, C. T., Hu, J. J., Chen, Y. S., Huang, C. H., and Lou, C. W. (2012). Property evaluation of Bletilla striata/polyvinyl alcohol nano fibers and composite dressings. J. Nanomater. 2012, 5. doi: 10.1155/2012/519516

Lin, Y. L., Chen, W. P., and Macabalang, A. D. (2005). Dihydrophenanthrenes from Bletilla formosana. Chem. Pharm. Bull. 53, 1111-1113. doi: 10.1002/ chin.200608189

Liou, M. J., and Wu, T. S. (2002). Triterpenoids from Rubia yunnanensis. J. Nat. Prod. 65, 1283-1287. doi: 10.1021/np020038k

Liu, B. S., and Huang, T. B. (2010). A novel wound dressing composed of nonwoven fabric coated with chitosan and herbal extract membrane for wound healing. Polym. Composite. 31, 1037-1046. doi: 10.1002/pc.20890

Liu, F. Q., Wang, Y. P., Han, D., Bi, Y. J., Li, H. B., Liu, G., et al. (2013). Extraction of Bletilla striata polysaccharide and its relative molecular mass determination and structure study. Chin. Tradit. Pat. Med. 35, 2291-2293. doi: 10.3969/j. issn.1001-1528.2013.10.055

Liu, M. X., Song, T. T., Ye, C., Zhang, Y., Shi, Y. H., and Dai, J. L. (2018). Inhibitory effect of Bletilla Striata polysaccharide on the proliferation of HepG2. AsiaPacific. Tradit. Med. 14, 11-13. doi: 10.11954/ytctyy. 201803005

Liu, R., Teng, X. J., He, J. F., Xiao, S. S., Yuan, Z. B., Li, X. J., et al. (2011). Partial splenic embolization using Bletilla striata particles for hypersplenism in cirrhosis: a prospective study. Am. J. Chin. Med. 39, 261-269. doi: 10.1142/ S0192415X11008804

Liu, S., Li, Y., Li, W. F., Xu, J. K., Li, F., and Du, H. (2016). Advances in studies on toxicity and modern toxicology of species in. Aconitum L. Chin. Tradit. Herbal. Drugs 47, 4095-4102. doi: 10.7501/j.issn.0253-2670.2016.22.027

Liu, X. X., Dong, L., Zhang, X. H., Dong, Y. X., Wang, A. M., Liao, S. G., et al. (2014). Quality evaluation of Bletillae Rhizoma based on hemostatic biopotency. Chin. J. Chin. Mater. Med. 39, 2051-2055. doi: 10.4268/cjcmm20141920

Lolmede, K., Campana, L., Vezzoli, M., Bosurgi, L., Tonlorenzi, R., Clementi, E., et al. (2009). Inflammatory and alternatively activated human macrophages attract vessel-associated stem cells, relying on separate HMGB1- and MMP-9dependent pathways. J. Exp. Med. 85, 779-787. doi: 10.1189/jlb.0908579

Lu, B., Xu, Y. M., Zhang, H. M., Li, T. J., and Qiu, Y. (2005). Effects of different extracts from Bletilla Colloid on rabbit platelet aggregation. Pharm. J. PLA. 21, 330-331. doi: 10.3969/j.issn.1008-9926.2005.05.004

Luo, Y., Diao, H. J., Xia, S. H., Dong, L., Chen, J. N., and Zhang, J. F. (2010). A physiologically active polysaccharide hydrogel promotes wound healing. J. Biomed. Mater. Res. A 94, 193-204. doi: 10.1002/jbm.a.32711

Ma, X. J., Cui, B. S., Han, S. W., and Li, S. (2017). Chemical constituents from tuber of Bletilla striata. Chin. J. Chin. Mater. Med. 42, 1578-1584. doi: 10.19540/j. cnki.cjcmm.2017.0051

Miller, E. R., Roberto, P. B., Darshan, D., Riemersma, R. A., Appel, L. J., and Eliseo, G. (2005). Meta-analysis: high-dosage vitamin E supplementation may increase all-cause mortality. Ann. Intern. Med. 142, 37-46. doi: 10.1016/j. accreview.2005.04.017

Mittler, R. (2002). Oxidative stress, antioxidants and stress tolerance. Trends Plant Sci. 7, 405-410. doi: 10.1016/S1360-1385(02)02312-9

Morita, H., Koyama, K., Sugimoto, Y., and Kobayashi, J. I. (2005). Antimitotic activity and reversal of breast cancer resistance protein-mediated drug resistance by stilbenoids from Bletilla striata. Bioorg. Med. Chem. Lett. 15, 1051-1054. doi: 10.1016/j.bmcl.2004.12.026

Park, J. E., Woo, K. W., Sang, U. C., Lee, J. H., and Kang, R. L. (2014). Two new cytotoxic spirostane-steroidal saponins from the roots of Bletilla striata. Helv. Chim. Acta 97, 56-63. doi: 10.1002/hlca.201300102

Peng, Q., Li, M., Xue, F., and Liu, H. J. (2014). Structure and immunobiological activity of a new polysaccharide from Bletilla striata. Carbohyd. Polym. 107, 119-123. doi: 10.1016/j.carbpol.2014.02.042

Qian, C. D., Jiang, F. S., Yu, H. S., Shen, Y., Fu, Y. H., Cheng, D. Q., et al. (2015). Antibacterial biphenanthrenes from the fibrous roots of Bletilla striata. J. Nat. Prod. 78, 939-943. doi: 10.1021/np501012n

Qian, J., Vossoughi, D., Woitaschek, D., Oppermann, E., Bechstein, W. O., Li, W. Y., et al. (2003). Combined transarterial chemoembolization and arterial administration of Bletilla striata in treatment of liver tumor in rats. World J. Gastroentero. 9, 50-54. doi: 10.1080/00365520310005712

Qiu, H. M., Ying, Z., Zhou, Q. X., and Shu, L. (2011). Regulatory effect of Bletilla striata polysaccharide on immune function of mice. Chin. J. Biologicals 24, 676-678. doi: 10.13200/j.cjb. 2011.06.57.qiuhm.010

Qu, Y., Li, C. X., Zhang, C., Zeng, R., and Fu, C. M. (2016). Optimization of infrared-assisted extraction of Bletilla striata polysaccharides based on response surface methodology and their antioxidant activities. Carbohyd. Polym. 148, 345-353. doi: 10.1016/j.carbpol.2016.04.081

Rhee, J. K., Kim, P. G., Baek, B. K., Lee, S. B., and Ahn, B. Z. (1982). Isolation of anthelmintic substance on clonorchis sinensis from tuber of Bletilla striata. Korean J. Parasitol. 20, 142. doi: 10.3347/kjp.1982.20.2.142

Ruiz, A., and Russell, S. J. (2012). A new paradigm in viral resistance. Cell Res. 22, 1515-1517. doi: 10.1038/cr.2012.139

Saito, N., Ku, M., Tatsuzawa, F., Lu, T. S., Yokoi, M., Shigihara, A., et al. (1995). Acylated cyanidin glycosides in the purple-red flowers of Bletilla striata. Phytochemistry 40, 1523-1529. doi: 10.1016/0031-9422(95)00479-Q

Shi, Y., Bing, Z., Lu, Y. Y., Qian, C. D., Yan, F., Fang, L. W., et al. (2017). Antiviral activity of phenanthrenes from the medicinal plant Bletilla striata against influenza A virus. BMC Complem. Altern. M. 17, 273-287. doi: 10.1186/s12906-017-1780-6

Song, Y., Zeng, R., Hu, L. L., Maffucci, K. G., Ren, X. D., and Qu, Y. (2017). In vivo wound healing and in vitro antioxidant activities of Bletilla striata phenolic extracts. Biomed. Pharmacother. 93, 451-461. doi: 10.1016/j.biopha.2017.06.079

Srinivasan, M., Sudheer, A. R., Pillai, K. R., Kumar, P. R., Sudhakaran, P. R., and Menon, V. P. (2006). Influence of ferulic acid on $\gamma$-radiation induced DNA damage, lipid peroxidation and antioxidant status in primary culture of isolated rat hepatocytes. Toxicology 228, 249-258. doi: 10.1016/j.tox.2006.09.004

Stintzing, F. C., and Carle, R. (2004). Functional properties of anthocyanins and betalains in plants, food, and in human nutrition. Trends Food Sci. Tech. 15, 19-38. doi: 10.1016/j.tifs.2003.07.004

Sun, A. J., Liu, J. Q., Pang, S. Q., Lin, J. S., and Xu, R. A. (2016a). Two novel phenanthraquinones with anti-cancer activity isolated from Bletilla striata. Bioorg. Med. Chem. Lett. 26, 2375-2379. doi: 10.1016/j.bmcl.2016.01.076

Sun, A. J., Pang, S. Q., and Wang, G. Q. (2016b). Chemical constituents from Bletilla striata and their anti-tumor activities. Chin. Pharmacol. J. 51, 101-104. doi: 10.11669/cpj.2016.02.005

Sun, A. J., Pang, S. Q., and Wang, G. Q. (2016c). Separation of chemical constituents from Bletilla striata and their antitumor activities. Chin. Pharmacol. J. 47, 35-38. doi: 10.1002/chin.201634198

Sun, J. T., Zhang, J. F., and Chen, Y. X. (2005). Embolization of carotid aneurysms in rabbit models using self-made liquid embolic agent from Bletillae Rhizoma. Chin. J. Minim. Invas. Neurosur. 10, 462-464. doi: 10.3969/j.issn.1009-122X.2005.10.011

Süntar, I., Akkol, E. K., Nahar, L., and Sarker, S. D. (2012). Wound healing and antioxidant properties: do they coexist in plants? Free Radical. Antioxid. 2, 1-7. doi: 10.5530/ax.2012.2.2.1

Takagi, S., Yamaki, M., and Inoue, K. (1983). Antimicrobial agents from Bletilla striata. Phytochemistry 22, 1011-1015. doi: 10.1016/0031-9422(83)85044-4

Tang, Y. F., Ruan, C. F., Ying, C., and Zhang, H. W. (2014). Research progress on chemical constituents and medical functions in plants of Bletilla Rchb. f. Chin. Tradit. Herbal. Drugs 45, 2864-2872. doi: 10.7501/j.issn.0253-2670.2014.19.025

Tao, Y., Tang, X. D., Li, Z. H., Zhang, L. Y., Zhang, B. P., and Du, N. (2015). Research overview of clinical application of TCM powders in digestive system diseases. Chin. J. Inf. Tradit. Chin. Med. 22, 128-130. doi: 10.3969/j. issn.1005-5304.2015.09.039

Tatsuzawa, F., Saito, N., Shigihara, A., Honda, T., Toki, K., Shinoda, K., et al. (2010). An acylated cyanidin 3,7-diglucoside in the bluish flowers of Bletilla striata 'Murasaki Shikibu' (Orchidaceae). J. Jpn. Soc. Hortic. Sci. 79, 215-220. doi: $10.2503 /$ jjshs 1.79 .215

Tsurusaki, M., and Murakami, T. (2015). Surgical and locoregional therapy of HCC: TACE. Liver Cancer 4, 165-175. doi: 10.1159/000367739

Venkatraja, B., Vanitha Malathy, V., Balasubramanian, E., Mohan, Rajendran, R., and Rammohan, R. (2012). Biopolymer and Bletilla striata herbal extract coated cotton gauze preparation for wound healing. J. Med. Sci. 12, 148-160. doi: 10.3923/jms.2012.148.160

Wang, A. M., Yan, Y., Lan, B., Liao, S. G., Wang, Y. L., and Li, Y. J. (2014). Simultaneous determination of nine chemical markers of Bletillae Rhizoma by ultra performance liquid chromatography. Chin. J. Chin. Mater. Med. 39, 2051-2055. doi: $10.4268 / \mathrm{cjcmm} 20141121$ 
Wang, C., Luo, W. F., Li, P. W., Li, S. D., Yang, Z. M., Zhang, H., et al. (2017). Preparation and evaluation of chitosan/alginate porous microspheres/Bletilla striata polysaccharide composite hemostatic sponges. Carbohyd. Polym. 174, 432-442. doi: 10.1016/j.carbpol.2017.06.112

Wang, C. M., Sun, J. T., Yi, L., Xue, W. H., Diao, H. J., Lei, D., et al. (2006). A polysaccharide isolated from the medicinal herb Bletilla striata induces endothelial cells proliferation and vascular endothelial growth factor expression in vitro. Biotechnol. Lett. 28, 539-543. doi: 10.1007/s10529-006-0011-X

Wang, C. X., Zhu, J. X., Ma, J. F., Yang, Y., and Cui, X. M. (2019). Functionalized Bletilla striata polysaccharide micelles for targeted intracellular delivery of Doxorubicin: in vitro and in vivo evaluation. Int. J. Pharm. 567, 118436-118446. doi: 10.1016/j.ijpharm.2019.06.027

Wang, G. H., Guo, X. Y., Wang, N. L., Zhang, J. C., Yang, M. S., and Yao, X. S. (2007). Study on cytotoxicity of four 9,10-dihydrophenanthrenes. Chin. Pharmacol. J. 42, 181-183. doi: 10.1631/jzus.2007.B0566

Wang, L. X., Han, G. X., Shu, Y., Liu, W. Y., and Zhang, W. D. (2001). Studies on chemical constituents of Bletilla striata(Thunb) Reichb.f. Chin. J. Chin. Mater. Med. 26, 690-692. doi: 10.3321/j.issn:1001-5302.2001.10.016

Wang, W., and Meng, H. (2015). Cytotoxic, anti-inflammatory and hemostatic spirostane-steroidal saponins from the ethanol extract of the roots of Bletilla striata. Fitoterapia 101, 12-18. doi: 10.1016/j.fitote.2014.11.005

Weng, X. G., Nie, S. Q., and Huang, L. Q. (2004). Determination of content changes of hypaconitine in preparations of aconite matching other herbs in "pinellia tuber, snakegourd fruit, fritillaria, japanese ampelopsis root and common bletilla tuber counteract aconite" by HPLC. Chin. Pharmacol. J. 39, 57-59. doi: $10.2500 / 105065898780182390$

Woo, K. W., Park, J. E., Choi, S. U., Kim, K. H., and Lee, K. R. (2014). Phytochemical constituents of Bletilla striata and their cytotoxic activity. Nat. Prod. Sci. 20, 91-94.

Xiao, S. J., Xu, D. L., Zhang, M. S., Linghu, L., Ding, L. S., Zhou, S. Y., et al. (2016). A novel phenanthrene-1,2-dione from Bletilla striata. Chin. J. Org. Chem. 36, 638. doi: $10.6023 /$ cjoc201509023

Xiao, S. J., Yuan, F. M., Zhang, M. S., Yu, S. Y., Li, J. D., Yi, X. D., et al. (2017). Three new 1-(p-hydroxybenzyl)phenanthrenes from Bletilla striata. J. Asian Nat. Prod. Res. 19, 140-144. doi: 10.1080/10286020.2016.1184254

Yamaki, M., Bai, L., Inoue, K., and Takagi, S. (1989). Biphenanthrenes from Bletilla striata. Phytochemistry 28, 3503-3505. doi: 10.1016/0031-9422(89)80373-5

Yamaki, M., Bai, L., Kato, T., Keiko, I., and Shuzo, T. (1993a). Three dihydrophenanthropyrans from Bletilla striata. Phytochemistry 32, 427-430. doi: 10.1016/S0031-9422(00)95008-8

Yamaki, M., Bai, L., Kato, T., Keiko, I., Shuzo, T., Yamagata, Y., et al. (1993b). Blespirol, a phenanthrene with a spirolactone ring from Bletilla striata. Phytochemistry 33, 1497-1498. doi: 10.1016/0031-9422(93)85119-C

Yamaki, M., Bai, L., Kato, T., Keiko, I., Takagi, S., Yamagata, Y., et al. (1992). Bisphenanthrene ethers from Bletilla striata. Phytochemistry 31, 3985-3987. doi: 10.1016/S0031-9422(00)97568-X

Yamaki, M., Bai,L., Keiko, I., and Shuzo, T. (1990). Benzylphenanthrenes from Bletilla striata. Phytochemistry 29, 2285-2287. doi: 10.1016/0031-9422(90)83053-4

Yamaki, M., Honda, C., Kato, T., Bai, L., and Takagi, S. (1997). The steroids and triterpenoids from Bletilla striata. Nat. Med. 51, 493.

Yamaki, M., Kato, T., Bai, L., Inoue, K., and Takagi, S. (1991). Methylated stilbenoids from Bletilla striata. Phytochemistry 30, 2759-2760. doi: 10.1016/0031-9422(91)85139-Q

Yamaki, M., Kato, T., Li, B., Inoue, K., and Takagi, S. (1993c). Phenanthrene glucosides from Bletilla striata. Phytochemistry 34, 535-537. doi: 10.1016/0031-9422(93)80041-P

Yan, Y., Guan, H. Y., Wang, A. M., Wang, Y. L., Li, Y. J., Liao, S. G., et al. (2014). Chemical constituents of Bletillae Rhizoma. Chin. J. Exp. Tradit. Med. Form. 20, 57-60. doi: 10.13422/j.cnki.syfjx.2014180057

Yang, C., Xia, T., Wang, C., Sun, H., Li, Y., Gong, Z., et al. (2019). Using the UPLCESI-Q-TOF-MS(E) method and intestinal bacteria for metabolite identification in the nonpolysaccharide fraction from Bletilla striata. Biomed. Chromatogr. 2019, e4637. doi: 10.1002/bmc.4637

Yang, L., Peng, C., Meng, C. W., He, C. J., Li, X. H., Guo, L., et al. (2014). A new macrolide and six cycloartane triterpenoids from the tubers of Bletilla striata. Biochem. Syst. Ecol. 57, 238-241. doi: 10.1016/j.bse.2014.08.020

Yang, N., Dang, S. S., Shi, J. J., Wu, F. P., Li, M., Zhang, X., et al. (2017). Caffeic acid phenethyl ester attenuates liver fibrosis via inhibition of TGF- $\beta 1 / \mathrm{Smad} 3$ pathway and induction of autophagy pathway. Biochem. Bioph. Res. Co. 486, 22-28. doi: 10.1016/j.bbrc.2017.02.057

Yang, X. Z., Tang, C. P., Zhao, P., Shu, G. W., and Mei, Z. N. (2012). Antimicrobial constituents from the tubers of Bletilla ochracea. Planta Med. 78, 606-610. doi: 10.1055/s-0031-1298264

Yu, H. S., Dai, B. L., Qian, C. D., Ding, Z. S., Jiang, F. S., Jin, B., et al. (2016). Antibacterial activity of chemical constituents isolated from fibrous roots of Bletilla striata. Chin. Med. Mat. 39, 544-547. doi: 10.13863/j. issn1001-4454.2016.03.019

Yu, L. H., Nie, X. Q., Pan, H. J., Ling, S., Zhang, D. D., and Bian, K. (2011). Diabetes mellitus ulcers treatment with Bletilla striata polysaccharide. Chin. J. Chin. Mater. Med. 36, 1487-1491. doi: 10.4268/cjcmm20111118

Yue, L., Wang, W., Wang, Y., Du, T., Shen, W. P., Tang, H. L., et al. (2016). Bletilla striata polysaccharide inhibits angiotensin II-induced ROS and inflammation via NOX4 and TLR2 pathways. Int. J. Biol. Macromol. 89, 376-388. doi: 10.1016/j.ijbiomac.2016.05.002

Yue, S. S., Tian, H. L., Li, L. M., Zhang, Y. X., Wang, X. H., and Han, J. G. (2003). Toxicity study of Bletilla gum. Chin. J. Exp. Tradit. Med. Form. 9, 63-64. doi: 10.3969/j.issn.1005-9903.2003.01.025

Zhang, B., Shi, Y., Zhou, F. M., Lu, Y. Y., and Cheng, D. Q. (2017a). Anti-influenza viruse activity of extracts from the tubes of Bletilla striata in vitro. Chin. Med. Mat. 40, 2930-2935. doi: 10.13863/j.issn1001-4454.2017.12.042

Zhang, C., Gao, F., Gan, S., He, Y. N., Chen, Z. J., Liu, X. W., et al. (2019a). Chemical characterization and gastroprotective effect of an isolated polysaccharide fraction from Bletilla striata against ethanol-induced acute gastric ulcer. Food Chem. Toxicol. 131, 110539. doi: 10.1016/j.fct.2019.05.047

Zhang, C., Zeng, R., Liao, Z. C., Fu, C. M., Luo, H., Yang, H. S., et al. (2017b). Bletilla striata micron particles function as a hemostatic agent by promoting rapid blood aggregation. Evid-based Compl. Alt. 2017, 1-8. doi: 10.1155/2017/5820405

Zhang, G. Y., Qian, J., Liu, X., Liu, Y. R., Wu, J., Huang, L., et al. (2019b). Interactions of self-assembled Bletilla Striata polysaccharide nanoparticles with bovine serum albumin and biodistribution of its docetaxel-loaded nanoparticles. Pharmaceutics 11, 43-64. doi: 10.3390/pharmaceutics11010043

Zhang, L., Ji, Y. X., Kang, Z. C., Lv, C. J., and Jiang, W. L. (2015). Protocatechuic aldehyde ameliorates experimental pulmonary fibrosis by modulating HMGB1/ RAGE pathway. Toxicol. Appl. Pharm. 283, 50-56. doi: 10.1016/j.taap.2015.01.001

Zhang, T., Zhuang, P. W., Lai, X. Y., Lu, Z. Q., Li, L. J., and Zhang, Y. J. (2013). Acute toxicity studies on compatibility of "pinellia, trichosanthes, fritillaria, ampelopsis, bletilla attack aconitum”. Chin. Tradit. Herbal. Drugs 44, 2442 2445. doi: 10.7501/j.issn.0253-2670.2013.17.019

Zhang, W. M., Ma, S. H., Gu, G. P., Shi, J. S., and Zhao, B. t. (2003). Studies on the toxicological assessment on skin safety of polysaccharide Gum of Bletilla striata (Thunb.) Reichb.f. Chin. Wild Plant Resour. 22, 59-61. doi: 10.3969/j. issn.1006-9690.2003.05.019

Zhao, F. F., Yang, X., Xu, D., Dong, L., Li, J., Wang, Y. L., et al. (2016). Hemostatic effect and mechanism of a non-polysaccharide fraction of Bletilla striata. Chin. Pharm. Bull. 32, 1121-1126. doi: 10.3969/j.issn.1001-1978.2016.08.018

Zhao, J. G., Feng, G. S., Kong, X. Q., Li, X., Li, M. H., and Cheng, Y. S. (2004). Changes of tumor microcirculation after transcatheter arterial chemoembolization:First pass perfusion MR imaging and Chinese ink casting in a rabbit model. World J Gastroentero. 10, 1415-1420. doi: 10.1360/972009-495

Zhao, W. H., Deng, Z. Y., Fan, Y. W., Jing, L., and Zheng, R. (2010). Antioxidant properties of ferulic acid in vitro. Food Sci. 31, 219-223. doi: 10.1360/972010-1292

Zhou, H., Jin, Y., Gu, C., Chen, Y., and Xia, J. (2019). Bletilla striata promotes the healing of enterocutaneous fistula: a case report. Medicine (Baltimore) 98, e16288. doi: 10.1097/MD.0000000000016288

Conflict of Interest: The authors declare that the research was conducted in the absence of any commercial or financial relationships that could be construed as a potential conflict of interest.

Copyright (c) $2019 \mathrm{Xu}$, Pan and Chen. This is an open-access article distributed under the terms of the Creative Commons Attribution License (CC BY). The use, distribution or reproduction in other forums is permitted, provided the original author(s) and the copyright owner(s) are credited and that the original publication in this journal is cited, in accordance with accepted academic practice. No use, distribution or reproduction is permitted which does not comply with these terms. 